\title{
Extensions of the asymptotic symmetry algebra of general relativity
}

\author{
Éanna É. Flanagan, ${ }^{a}$ Kartik Prabhu ${ }^{b}$ and Ibrahim Shehzad ${ }^{a}$ \\ ${ }^{a}$ Department of Physics, Cornell University, \\ Ithaca, NY 14853, U.S.A. \\ ${ }^{b}$ Cornell Laboratory for Accelerator-based Sciences and Education (CLASSE), Cornell University, \\ Ithaca, NY 14853, U.S.A. \\ E-mail: eef3@cornell.edu, kartikprabhu@cornell.edu, is354@cornell.edu
}

AbSTRACT: We consider a recently proposed extension of the Bondi-Metzner-Sachs algebra to include arbitrary infinitesimal diffeomorphisms on a 2-sphere. To realize this extended algebra as asymptotic symmetries, we work with an extended class of spacetimes in which the unphysical metric at null infinity is not universal. We show that the symplectic current evaluated on these extended symmetries is divergent in the limit to null infinity. We also show that this divergence cannot be removed by a local and covariant redefinition of the symplectic current. This suggests that such an extended symmetry algebra cannot be realized as symmetries on the phase space of vacuum general relativity at null infinity, and that the corresponding asymptotic charges are ill-defined. However, a possible loophole in the argument is the possibility that symplectic current may not need to be covariant in order to have a covariant symplectic form. We also show that the extended algebra does not have a preferred subalgebra of translations and therefore does not admit a universal definition of Bondi 4-momentum.

Keywords: Classical Theories of Gravity, Gauge Symmetry, Space-Time Symmetries

ARXIV EPRINT: 1910.04557 


\section{Contents}

1 Introduction and summary 1

1.1 Notation and conventions 4

2 Asymptotic flatness at null infinity and the BMS algebra 4

2.1 Definition and properties of asymptotic flatness at null infinity 4

2.2 Review of derivation of the Bondi-Metzner-Sachs symmetry algebra 6

3 An extended field configuration space and extended algebra $\quad 8$

$\begin{array}{lll}3.1 & \text { Extended field configuration space } & 9\end{array}$

$\begin{array}{lll}3.2 & \text { Extended algebra } & 9\end{array}$

4 The symplectic current of general relativity at null infinity 10

$\begin{array}{lll}4.1 & \text { The symplectic current for general perturbations } & 11\end{array}$

$\begin{array}{ll}4.2 & \text { Divergence of the symplectic current on the extended phase space }\end{array}$

$\begin{array}{lll}4.3 & \text { Ambiguities in the symplectic current } & 14\end{array}$

5 Other issues 17

$\begin{array}{ll}5.1 \text { Existence of Bondi four-momentum } & 17\end{array}$

$\begin{array}{ll}5.2 \text { Choice of field configuration space } & 18\end{array}$

$\begin{array}{llr}6 & \text { Discussion and conclusions } & 18\end{array}$

A Metric on $\mathscr{I}$ and conformal factor in a neighborhood can be chosen to be universal 19

B The extended BMS algebra does not contain any preferred translation subalgebra

\section{Introduction and summary}

The asymptotic symmetry group at null infinity of asymptotically-flat spacetimes in general relativity is normally considered to be the infinite-dimensional Bondi-Metzner-Sachs (BMS) group. Associated with the Lie algebra of the BMS group there are an infinite number of charges and fluxes (due to gravitational radiation) at null infinity [1-8]. Recently, these charges and fluxes have been related to soft graviton theorems [9-14], gravitational memory effects $[9,10,15-19]$ and potentially black hole information loss [20-23].

There have been attempts to extend these relations to include the subleading soft theorems, in particular, the one proposed by Cachazo and Strominger in [24], by enlarging the 
gravitational phase space to give additional asymptotic symmetries. To understand the nature of these proposed enlargements consider, briefly, the structure of the BMS algebra (see section 2 for details). The BMS algebra contains an infinite-dimensional extension of the usual translations - known as supertranslations - as well as (many equivalent subalgebras of) infinitesimal Lorentz transformations. These Lorentz transformations are conformal Killing fields on 2-sphere cross-sections of null infinity which are smooth everywhere. It was proposed by Barnich and Troessaert [25, 26] (see also [27]) that the BMS algebra should be extended to include the entire infinite-dimensional Virasoro algebra, which consists of all local conformal Killing fields on a 2 -sphere. ${ }^{1}$ The vector fields in the Virasoro algebra which are not Lorentz vector fields are necessarily singular at isolated points on a 2 -sphere. An alternative proposal by Campiglia and Laddha [29, 30] was to extend the Lorentz transformations by including all smooth infinitesimal diffeomorphisms on a 2-sphere. The conservation law (i.e. Ward identity) for the charges at null infinity corresponding to such extensions is then claimed to be equivalent to the subleading soft theorem of [24].

In this paper, we use the symplectic formalism for general relativity to investigate whether such extensions of the asymptotic symmetries algebra have well-defined charges at null infinity. Since the Virasoro vector fields are in general singular, it is tricky to apply the usual symplectic formalism to such symmetries. Instead, we analyze the second extension of smooth 2-sphere diffeomorphisms [29, 30] mentioned above.

The main quantity of interest in our analysis is the symplectic current derived from the Lagrangian of general relativity (section 4). The symplectic current is a local and covariant 3 -form $\boldsymbol{\omega}\left(\tilde{g} ; \delta_{1} \tilde{g}, \delta_{2} \tilde{g}\right)$ which is an antisymmetric bilinear in two perturbations of the metric $\delta_{1} \tilde{g}_{a b}$ and $\delta_{2} \tilde{g}_{a b}$ (we have used a "tilde" to denote quantities in the physical spacetime as opposed to ones in the Penrose conformal completion). If suitable asymptotic conditions are satisfied then the symplectic current has a finite limit to null infinity. Then, the integral of the symplectic current over null infinity gives a symplectic form on the phase space of general relativity. If one of the perturbations, say $\delta_{2} \tilde{g}_{a b}$, is taken to be the perturbation generated by some asymptotic symmetry, then the symplectic form gives an expression for the generator of that symmetry on phase space. Note that the crucial aspect of the above is that the symplectic current must have a finite limit to null infinity, otherwise the generator would not be defined.

It is well-known that for asymptotically-flat spacetimes the symmetries in the usual BMS algebra have well-defined generators in the sense described above $[7,8]$. We are interested in whether generators corresponding to the extension of the BMS algebra by all diffeomorphisms of a 2-sphere exist. We show that the answer is no: the symplectic current of general relativity diverges in the limit to null infinity, in general, when one of the perturbations is generated by an extended BMS symmetry (which is not a BMS symmetry). This divergence was also previously encountered in the computations of Compère, Fiorucci and Ruzziconi [28]. This divergence suggests that the generators of such extended symmetries

\footnotetext{
${ }^{1}$ These symmetries are often called superrotations $[25,26]$, although more recently that term has come to be used for the smooth infinitesimal diffeomorphisms on the 2-sphere [23]. Another terminology for the smooth diffeomorphisms is super-Lorentz transformations, with the odd parity ones being called superrotations and the even parity ones being called superboosts [28].
} 
may not exist on the phase space at null infinity, and the corresponding charges and fluxes may be also ill-defined.

A loophole in this argument is that one can exploit an ambiguity in the symplectic current to render it finite in the limit to null infinity (see ref. [31] for a general discussion of such renormalization in a different context). The ambiguity is of the form $\boldsymbol{\omega} \rightarrow \boldsymbol{\omega}+$ $d\left[\delta_{1} \boldsymbol{Y}\left(\tilde{g} ; \delta_{2} \tilde{g}\right)-(1 \leftrightarrow 2)\right]$, for some two-form $\boldsymbol{Y}$ which constructed out of the dynamical fields and their variations [8]. Recently, Compère, Fiorucci and Ruzziconi have shown that one can indeed obtain a finite symplectic current using this method, and they find expressions for charges corresponding to all the symmetries of the extended algebra, including the general 2-sphere diffeomorphisms [28].

However, as noted by the authors themselves, their prescription relies on a particular choice of coordinates with the result that the two form $\boldsymbol{Y}$ and the final, finite symplectic current are not local, covariant function of the dynamical fields. Thus, it is not clear that the expressions obtained in ref. [28] for charges are unique. For instance, if one repeated the construction using Newman-Unti coordinates instead of Bondi coordinates, it is not clear if equivalent results would be obtained. We will show that one cannot eliminate the divergences in the symplectic current by exploiting the ambiguity in a local and covariant manner.

This result suggests that the general 2-sphere diffeomorphisms do not give rise to well defined charges and fluxes. However, a possible loophole is that requiring that all the quantities in the construction be local and covariant [8] is too strong a restriction, and instead one should only impose this requirement on physically measurable quantities. For example it might be possible that the presymplectic form (obtained by integrating the presymplectic current over a Cauchy surface) may be independent of the arbitrary choice of coordinate system used in ref. [28], despite the fact that the presymplectic current $\boldsymbol{\omega}$ does depend on this choice. It would be interesting to investigate this possibility further, but we do not do so in this paper.

The remainder of this paper is organized as follows. In section 2, we review the definition of asymptotic flatness and show how the BMS algebra emerges as the asymptotic symmetry algebra of asymptotically-flat spacetimes. In section 3, we consider the extended phase space proposed in [30] which leads to an extension of the BMS algebra to include arbitrary infinitesimal diffeomorphisms of a 2 -sphere. In section 4 , we show that the symplectic current evaluated on these extended symmetries diverges in the limit to null infinity. We also show that any local and covariant ambiguities in the symplectic current cannot get rid of this divergent behavior. We consider other issues associated with this extension of the BMS algebra in section 5 . We end by summarizing our main conclusions in section 6. In appendix A, we construct suitable coordinate systems near $\mathscr{I}$ and show that the conformal factor and the unphysical metric at null infinity can be chosen universally in the class of asymptotically-flat spacetimes. In appendix B, we show that the extension of the BMS algebra by all diffeomorphisms of a 2 -sphere does not contain any preferred translation subalgebra. 


\subsection{Notation and conventions}

We follow the conventions of Wald [32] throughout. Tensors on spacetime will be denoted by Latin indices $a, b, c, \ldots$. We will frequently use an index-free notation for differential forms and denote then by a bold-face, e.g. $\boldsymbol{\omega} \equiv \omega_{a b c}$ is the 3 -form symplectic current. Tensors on the physical spacetime will be denoted by a "tilde" while those on the conformal completion (unphysical spacetime) will not have a "tilde", e.g. $\tilde{g}_{a b}$ is the physical metric while $g_{a b}$ is the unphysical metric in the conformal completion. Indices on unphysical, unbarred quantities will be raised and lowered with the unphysical metric, for example $n_{a} n^{a}=g^{a b} n_{a} n_{b}$.

\section{Asymptotic flatness at null infinity and the BMS algebra}

In this section, we review the definition of asymptotically-flat spacetimes and show how the BMS algebra arises as the asymptotic symmetry algebra at null infinity.

\subsection{Definition and properties of asymptotic flatness at null infinity}

A physical spacetime $\left(\tilde{M}, \tilde{g}_{a b}\right)$, satisfying the vacuum ${ }^{2}$ Einstein equation $\tilde{G}_{a b}=0$, is asymptotically-flat at null infinity if there exists another unphysical spacetime $\left(M, g_{a b}\right)$ with a boundary $\mathscr{I}=\partial M$ and an embedding of $\tilde{M}$ into $M,{ }^{3}$ such that

1. there exists and a smooth function $\Omega$ (the conformal factor) on $M$ satisfying

$$
\begin{aligned}
\Omega & =0 \text { on } \mathscr{I}, \quad \nabla_{a} \Omega \text { is nowhere vanishing on } \mathscr{I}, \\
g_{a b} & =\Omega^{2} \tilde{g}_{a b} \text { is smooth on } M \text { including at } \mathscr{I} .
\end{aligned}
$$

2. $\mathscr{I}$ is topologically $\mathbb{R} \times \mathbb{S}^{2}$.

3. Defining the vector field

$$
n_{a}=\nabla_{a} \Omega,
$$

then the vector field $\omega^{-1} n^{a}$ is complete on $\mathscr{I}$ for any smooth function $\omega$ on $M$ such that $\omega>0$ on $M$ and $\nabla_{a}\left(\omega^{4} n^{a}\right)=0$ on $\mathscr{I}{ }^{4}$

For detailed expositions on the motivations for this definition, we refer the reader to refs. [5, 32]. Note that the smoothness conditions on the unphysical spacetime can be significantly weakened - we can allow $M$ to have a $C^{3}$-differential structure and $g_{a b}$ to be twicedifferentiable at the boundary $\mathscr{I}$.

\footnotetext{
${ }^{2}$ For non-vacuum spacetimes, the definition of asymptotic flatness includes a fourth condition, that the physical stress-energy tensor $\tilde{T}_{a b}$ satisfies$$
\tilde{T}_{a b}=\Omega^{2} T_{a b}
$$

for some tensor $T_{a b}$ which is smooth on $M$ including at $\mathscr{I}$. All of the results in this paper generalize to the non-vacuum case, except for the discussion of the presymplectic current in section 4, which is specialized to vacuum general relativity.

${ }^{3}$ We use the standard convention whereby the physical spacetime $\tilde{M}$ is identified with its image in $M$ under the embedding.

${ }^{4}$ Since we will primarily be interested in the asymptotic symmetry algebra, and not the symmetry group, we will not need the completeness condition on $\mathscr{I}$.
} 
Using the conformal transformation relating the unphysical Ricci tensor $R_{a b}$ to the physical Ricci tensor $\tilde{R}_{a b}$ (see appendix D of [32]), the vacuum Einstein equation can be written as

$$
S_{a b}=-2 \Omega^{-1} \nabla_{(a} n_{b)}+\Omega^{-2} n^{c} n_{c} g_{a b},
$$

where $S_{a b}$ is given by

$$
S_{a b}=R_{a b}-\frac{1}{6} R g_{a b} .
$$

It follows immediately from eq. (2.4) and from the assumed smoothness of $\Omega$ and of the unphysical metric $g_{a b}$ at $\mathscr{I}$ that $\left.n_{a} n^{a}\right|_{\mathscr{I}}=0$. Hence $\mathscr{I}$ is a smooth null hypersurface in $M$ with normal $n_{a}=\nabla_{a} \Omega$ and the vector field $n^{a}=g^{a b} n_{b}$ is a null geodesic generator of $\mathscr{I}$.

Next, we write $n^{a} n_{a}=\chi \Omega$, where $\chi$ extends smoothly to $\mathscr{I}$, so that eq. (2.4) yields on $\mathscr{I}$ that

$$
2 \nabla_{(a} n_{b)}=\chi g_{a b}
$$

Under a change of the conformal factor of the form

$$
\Omega \mapsto \omega \Omega, \quad g_{a b} \mapsto \omega^{2} g_{a b},
$$

where $\omega$ is smooth in $M$ and is nowhere vanishing on $\mathscr{I}$, we have that $\chi$ transforms on $\mathscr{I}$ as $\chi \rightarrow\left(\chi+2 £_{n} \ln \omega\right) / \omega$. Hence we can choose $\omega$ to make $\chi=0[5,32]$, which yields from eq. (2.6) the Bondi condition

$$
\left.\nabla_{a} n_{b}\right|_{\mathscr{I}}=\left.\nabla_{a} \nabla_{b} \Omega\right|_{\mathscr{I}}=0
$$

as well as

$$
n_{a} n^{a}=O\left(\Omega^{2}\right)
$$

The remaining freedom in the conformal factor is of the form eq. (2.7) with

$$
\left.\omega\right|_{\mathscr{I}}>0,\left.\quad £_{n} \omega\right|_{\mathscr{I}}=0 .
$$

Let $q_{a b}$ the pullback of $g_{a b}$ to $\mathscr{I}$. This defines a degenerate metric on $\mathscr{I}$ such that

$$
q_{a b} n^{b}=0, \quad £_{n} q_{a b}=0,
$$

where the second condition follows from eq. (2.8). Thus, $q_{a b}$ defines a Riemannian metric on the space of generators of $\mathscr{I}$ which is diffeomorphic to $\mathbb{S}^{2}$.

A priori, the conformal completion depends on the physical spacetime $\left(\tilde{M}, \tilde{g}_{a b}\right)$ under consideration. However, if $\left(M, g_{a b}, \Omega\right)$ and $\left(M^{\prime}, g_{a b}^{\prime}, \Omega^{\prime}\right)$ are the unphysical spacetimes corresponding to any two asymptotically-flat physical spacetimes, then $M^{\prime}$ can be identified with $M$ using a diffeomorphism such that $\mathscr{I}^{\prime}$ maps to $\mathscr{I}, \Omega^{\prime}=\Omega$ in a neighborhood of $\mathscr{I}$ and $\left.g_{a b}^{\prime}\right|_{\mathscr{I}}=\left.g_{a b}\right|_{\mathscr{I}}[6]$. This can be shown by setting up a suitable geometrically-defined coordinate system in a neighborhood of $\mathscr{I}$ and identifying the two unphysical spacetimes in these coordinates; we defer the details to appendix A. Here we emphasize that the choice of coordinate system used is largely irrelevant. The only essential ingredients used in the identification are 
1. $\mathscr{I}$ is a null smooth surface in the unphysical spacetime $M$.

2. The freedom in the choice of the conformal factor $\Omega$ given by eq. (2.10).

3. The space of null generators of $\mathscr{I}$ is topologically $\mathbb{S}^{2}$ and thus has a unique conformal class of metrics up to diffeomorphisms.

As discussed above, the first two ingredients follow directly from the smoothness requirements in the definition of asymptotic flatness and the Einstein equation at $\mathscr{I}$. The third fact is a special case of the uniformization theorem (for instance see Ch. 8 [33]) and plays a crucial role on $\mathscr{I}^{5}{ }^{5}$ As we will show below this fact leads directly to the BMS algebra at $\mathscr{I}$ with the Lorentz algebra being a subalgebra (instead of all diffeomorphisms of $\mathbb{S}^{2}$ ). Further, it is also essential in the definition of a News tensor characterizing the presence of radiation at $\mathscr{I}$ (see Theorem 5 of $[5]$ ). ${ }^{6}$

As a result of this identification, we can work on a single manifold $M$ with boundary $\mathscr{I}$ and treat $\Omega$ and $\left.g_{a b}\right|_{\mathscr{I}}$ as universal within the entire class of asymptotically-flat spacetimes, in the sense that they can be chosen to be independent of the choice of the physical spacetime. Specifically, fix a metric $g_{0} a b$ on $\mathscr{I}$ and a conformal factor $\Omega_{0}$ in a neighborhood $\mathcal{N}$ of $\mathscr{I}$, and define the field configuration space ${ }^{7}$

$$
\Gamma_{0}=\left\{\left(M, g_{a b}, \Omega\right) \mid g_{a b \mid \mathscr{I}}=g_{0 a b \mid \mathscr{I}}, \quad \Omega=\Omega_{0} \text { on } \mathcal{N},\left.\quad \nabla_{a} \nabla_{b} \Omega\right|_{\mathscr{I}}=0\right\}
$$

Note that we include the Bondi condition (2.8) in this definition. Not all asymptotically flat unphysical metrics lie in $\Gamma_{0}$, but for each one there corresponds an element of $\Gamma_{0}$ related to it by a diffeomorphism and a conformal rescaling of the form (2.7), as we show in appendix A.

\subsection{Review of derivation of the Bondi-Metzner-Sachs symmetry algebra}

The asymptotic symmetries at $\mathscr{I}^{+}$of the field configuration space $\Gamma_{0}$ are the infinitesimal diffeomorphisms generated by vector fields $\xi^{a}$ in $M$ which extend smoothly to $\mathscr{I}$, and whose pullbacks preserve the asymptotic flatness conditions and map $\Gamma_{0}$ into itself, modded out by the trivial diffeomorphisms whose asymptotic charges vanish [8].

Consider a one-parameter family of asymptotically-flat physical metrics $\tilde{g}_{a b}(\lambda)$ where $\tilde{g}_{a b}=\tilde{g}_{a b}(\lambda=0)$ is some chosen background spacetime. Define the physical metric perturbation $\tilde{\gamma}_{a b}$ around the background $\tilde{g}_{a b}$ by

$$
\tilde{\gamma}_{a b}=\delta \tilde{g}_{a b}=\left.\frac{d}{d \lambda} \tilde{g}_{a b}(\lambda)\right|_{\lambda=0}
$$

We will use " $\delta$ " to denote perturbations of other quantities defined in a similar way.

\footnotetext{
${ }^{5}$ The uniformization theorem is a global result depending on the topology of the 2-dimensional space. Locally, all metrics of a particular signature on a 2-surface are conformally-equivalent, Problem 2 Ch. 3 [32].

${ }^{6}$ Even in spacetime dimensions $d>4$, to have well-defined Bondi mass and News tensor it appears essential to additionally assume that the metric $q_{a b}$ on the $(d-2)$-dimensional space of generators of $\mathscr{I}$ is conformal to a compact space of constant curvature [16, 34, 35].

${ }^{7}$ Sometimes called the pre-phase space or space of field histories [36].
} 
Now let $g_{a b}(\lambda)$ and $\Omega(\lambda)$ be one-parameter family of unphysical metrics and conformal factors corresponding to the conformal completions of the physical metrics $\tilde{g}_{a b}(\lambda)$. As discussed above, since the conformal factor $\Omega$ is universal we have $\delta \Omega=0$ and $\delta n_{a}=0$. The unphysical metric perturbation is then

$$
\delta g_{a b}=\gamma_{a b}=\Omega^{2} \tilde{\gamma}_{a b}
$$

where $\gamma_{a b}$ is smooth on $M$ and extends smoothly to $\mathscr{I}$. Since the unphysical metric at $\mathscr{I}$ is universal, we have that

$$
\left.\delta g_{a b}\right|_{\mathscr{I}}=\left.\gamma_{a b}\right|_{\mathscr{I}}=0 \Longrightarrow \gamma_{a b}=\Omega \tau_{a b},
$$

for some tensor $\tau_{a b}$ which extends smoothly to $\mathscr{I}$. Further, perturbing the Bondi condition (2.8) we can show that [8]

$$
\tau_{a b} n^{b}=\Omega \tau_{a},
$$

for some $\tau_{a}$ which extends smoothly to $\mathscr{I}$. Thus, the unphysical metric perturbations $\gamma_{a b}$ tangent to the field configuration space $\Gamma_{0}$ of eq. (2.12) satisfy

$$
\begin{aligned}
\gamma_{a b} & =\Omega \tau_{a b}, \\
\gamma_{a b} n^{b} & =\Omega^{2} \tau_{a} .
\end{aligned}
$$

Now consider the physical metric perturbation $£_{\xi} \tilde{g}_{a b}$ corresponding to an infinitesimal diffeomorphism generated by a vector field $\xi^{a}$. The corresponding unphysical metric perturbation is

$$
\gamma_{a b}^{(\xi)}=\Omega^{2} £_{\xi} \tilde{g}_{a b}=£_{\xi} g_{a b}-2 \Omega^{-1} n_{c} \xi^{c} g_{a b} .
$$

For $\xi^{a}$ to be a representative of an infinitesimal asymptotic symmetry, the perturbation (2.18) must satisfy the conditions (2.17). First, since $\gamma_{a b}^{(\xi)}$ is smooth at $\mathscr{I}$, we have from eq. (2.18) that $\left.n_{a} \xi^{a}\right|_{\mathscr{I}}=0$, that is, $\xi^{a}$ must be tangent to $\mathscr{I}$. We define the function $\alpha_{\xi}$ by

$$
n_{a} \xi^{a}=\Omega \alpha_{(\xi)},
$$

where $\alpha_{(\xi)}$ extends smoothly to $\mathscr{I}$. This yields

$$
\gamma_{a b}^{(\xi)}=£_{\xi} g_{a b}-2 \alpha_{(\xi)} g_{a b},
$$

and contracting with $n^{a} n^{b}$ and using eqs. (2.17) and (2.9) gives that

$$
n^{a} n^{b} \nabla_{a} \xi_{b}=O\left(\Omega^{2}\right) .
$$

Next, contracting eq. (2.20) with $n^{b}$ and using eqs. (2.8) and (2.19) gives

$$
n^{b} \gamma_{a b}^{(\xi)}=n^{b} \nabla_{b} \xi_{a}-\xi^{b} \nabla_{b} n_{a}-\alpha_{(\xi)} n_{a}+\Omega \nabla_{a} \alpha_{(\xi)},
$$

where we have used $\nabla_{a} n_{b}=\nabla_{b} n_{a}$ from eq. (2.3). From eqs. (2.17) the left-hand-side of eq. (2.22) must vanish at $\mathscr{I}$, which gives

$$
\left.£_{\xi} n^{a}\right|_{\mathscr{I}}=-\left.\alpha_{(\xi)} n^{a}\right|_{\mathscr{I}}
$$


Similarly the constraint (2.17b) gives using eqs. (2.3), (2.9), (2.19), (2.21) and (2.22)

$$
n^{a} n^{b} \gamma_{a b}^{(\xi)}=\left.O\left(\Omega^{2}\right) \Longrightarrow £_{n} \alpha_{(\xi)}\right|_{\mathscr{I}}=0 .
$$

Finally, the pullback of eq. (2.20) to $\mathscr{I}$ implies that

$$
\left.\gamma_{a b}^{(\xi)}\right|_{\mathscr{I}}=\left.0 \Longrightarrow £_{\xi} q_{a b}\right|_{\mathscr{I}}=2 \alpha_{(\xi)} q_{a b}
$$

Thus, representatives of asymptotic symmetries for $\Gamma_{0}$ on $\mathscr{I}$ are vector fields $\xi^{a}$ which are tangent to $\mathscr{I}$ and satisfy on $\mathscr{I}$

$$
\begin{aligned}
& £_{\xi} n^{a}=-\alpha_{(\xi)} n^{a}, \\
& £_{\xi} q_{a b}=2 \alpha_{(\xi)} q_{a b},
\end{aligned}
$$

where the function $\alpha_{(\xi)}$ is smooth and

$$
£_{n} \alpha_{(\xi)}=0
$$

on $\mathscr{I}$.

Next we need to mod out by trivial infinitesimal diffeomorphisms for which all boundary charges vanish. For the case of vacuum general relativity, the trivial vector fields $\xi^{a}$ are those which vanish on $\mathscr{I}[8]$, and so it follows that the symmetry algebra consists of intrinsic vector fields $\xi^{a}$ on $\mathscr{I}$ which satisfy the conditions (2.26) and (2.27) on $\mathscr{I}$. These conditions are the familiar ones defining the BMS algebra $\mathfrak{b}[5,7]$.

Finally we review some of the properties of this algebra. Consider vector fields of the form $\left.\xi^{a}\right|_{\mathscr{I}}=f n^{a}$ for which $\left.£_{n} f\right|_{\mathscr{I}}=0$. It follows from eqs. (2.9) and (2.19) that we have $\left.\alpha_{(\xi)}\right|_{\mathscr{I}}=0$. It is easy to verify that vector fields of this form generate an infinitedimensional abelian subalgebra $\mathfrak{s}$ of $\mathfrak{b}$. Further, this subalgebra is invariant in the sense that the Lie bracket of any element of $\mathfrak{b}$ with any element of $\mathfrak{s}$ is again in $\mathfrak{s}$, that is $\mathfrak{s}$ is a Lie ideal of $\mathfrak{b}$. This is the subalgebra of supertranslations. From eq. (2.26b), the factor algebra $\mathfrak{b} / \mathfrak{s}$ is isomorphic to the algebra of smooth conformal Killing fields of $q_{a b}$ on $\mathbb{S}^{2}$. Since the conformal class of metrics on $\mathbb{S}^{2}$ is unique up to diffeomorphisms, the algebra of smooth conformal Killing fields of any $q_{a b}$ is isomorphic to the algebra of smooth conformal Killing fields of the unit-metric on $\mathbb{S}^{2}$, that is the Lorentz algebra $\mathfrak{s o}(1,3)$. Thus, the BMS algebra has the semi-direct structure $\mathfrak{b}=\mathfrak{s o}(1,3) \ltimes \mathfrak{s}$. The BMS algebra also contains a unique 4-dimensional Lie ideal $\mathfrak{t}$ which can be interpreted as translations (see [3] or Theorem 6 of [5], also appendix B below). The presence of this preferred subalgebra $t$ implies that the Bondi 4-momentum at $\mathscr{I}$ is unambiguously defined.

\section{An extended field configuration space and extended algebra}

In this section we show that by weakening the universal structure near $\mathscr{I}$ - equivalently, by extending the class of allowed metrics — one obtains a bigger asymptotic symmetry algebra at null infinity which includes all the smooth diffeomorphisms of a 2 -sphere. This is the algebra proposed by Campiglia and Laddha $[29,30]$. 


\subsection{Extended field configuration space}

It is clear from the preceding section that to obtain any extension of the BMS algebra, one must enlarge the class of metrics under consideration. One option might be to suitably weaken the definition of asymptotic flatness. An alternative approach, which we follow here, is to enlarge the definition (2.12) of the field configuration space by relaxing the requirement that the unphysical metric evaluated on $\mathscr{I}$ be universal. The motivation for this enlargement is questionable, since the new metrics that are being added are related to metrics already included in the space $\Gamma_{0}$ by diffeomorphisms and by the conformal transformations (2.10). This issue is discussed further in section 5.2 below. Nevertheless, we shall proceed and consider the extended class of metrics proposed by Campiglia and Laddha [30].

In the definition of the extended field configuration space, we will continue to require that the unphysical metric $g_{a b}$ be smooth at $\mathscr{I}$. We will also continue to choose the conformal factor $\Omega$ so that the Bondi condition (2.8) holds. Now, if we are given an unphysical spacetime $\left(M, g_{a b}, \Omega\right)$, we can define tensors $\hat{n}^{a}$ and $\bar{\varepsilon}_{a b c}$ intrinsic to $\mathscr{I}$ by

$$
\begin{aligned}
\hat{n}^{a} & =\left.g^{a b} \nabla_{a} \Omega\right|_{\mathscr{I}}, \\
\left.\varepsilon_{a b c d}\right|_{\mathscr{I}} & =\left.4 \varepsilon_{[a b c} n_{d]}\right|_{\mathscr{I}},
\end{aligned}
$$

and by defining $\bar{\varepsilon}_{a b c}$ to be the pullback of $\varepsilon_{a b c}$ to $\mathscr{I} .{ }^{8}$ It follows from the Bondi condition (2.8) that

$$
£_{\hat{n}} \bar{\varepsilon}_{a b c}=0 .
$$

We now fix a choice of tensors $\hat{n}_{0}^{a}, \bar{\varepsilon}_{0 a b c}$ obtained in this way, fix a choice of conformal factor $\Omega_{0}$ on a neighborhood $\mathcal{N}$ of $\mathscr{I}$, and define the extended field configuration space $\Gamma_{\text {ext }}$ to be [compare eq. (2.12)]

$$
\Gamma_{\text {ext }}=\left\{\left(M, g_{a b}, \Omega\right) \mid \hat{n}^{a}=\hat{n}_{0}^{a}, \quad \bar{\varepsilon}_{a b c}=\bar{\varepsilon}_{0 a b c}, \quad \Omega=\Omega_{0} \text { on } \mathcal{N},\left.\quad \nabla_{a} \nabla_{b} \Omega\right|_{\mathscr{I}}=0\right\} .
$$

This is the definition proposed by Campiglia and Laddha [30], in which the 3 -volume form $\bar{\varepsilon}_{a b c}$ and the normal $\hat{n}^{a}$ at $\mathscr{I}$ are universal. Note that $\Gamma_{0}$ is a proper subset of $\Gamma_{\text {ext }}$, if we choose the fields $\hat{n}_{0}^{a}$ and $\bar{\varepsilon}_{0 a b c}$ to be those associated with $\left.g_{0 a b}\right|_{\mathscr{I}}$.

\subsection{Extended algebra}

We now derive the form of the symmetry algebra for the field configuration space (3.3). Since the fields $\bar{\varepsilon}_{a b c}$ and $\hat{n}^{a}$ are universal their perturbations must vanish, so

$$
\begin{aligned}
\left.\delta \bar{\varepsilon}_{a b c}\right|_{\mathscr{I}}=\left.0 \Longrightarrow g^{a b} \gamma_{a b}\right|_{\mathscr{I}} & =0 \\
\left.\delta n^{a}\right|_{\mathscr{I}}=0 & \Longrightarrow g^{a b} \gamma_{a b}=\Omega \sigma, \\
a b & n^{b}=\Omega \chi_{a},
\end{aligned}
$$

for some fields $\sigma$ and $\chi_{a}$ which extend smoothly to $\mathscr{I}$. Perturbing the Bondi condition (2.8) we have

$$
\left.\delta\left(\nabla_{a} n_{b}\right)\right|_{\mathscr{I}}=\left.0 \Longrightarrow n^{c} \nabla_{c} \gamma_{a b}\right|_{\mathscr{I}}=2 n_{(a} \chi_{b)},
$$

\footnotetext{
${ }^{8}$ Note that $\varepsilon_{a b c}$ is ambiguous up to $\varepsilon_{a b c} \mapsto \varepsilon_{a b c}+\alpha_{[a b} n_{c]}$ but this does not affect the pullback $\bar{\varepsilon}_{a b c}$.
} 
and taking the trace and using eq. (3.4) gives

$$
\left.n^{a} \chi_{a}\right|_{\mathscr{I}}=0
$$

Thus, the unphysical metric perturbations $\gamma_{a b}$ in the extended class satisfy

$$
g^{a b} \gamma_{a b}=\Omega \sigma, \quad \gamma_{a b} n^{b}=\Omega \chi_{a},\left.\quad n^{a} \chi_{a}\right|_{\mathscr{I}}=0,\left.\quad n^{c} \nabla_{c} \gamma_{a b}\right|_{\mathscr{I}}=2 n_{(a} \chi_{b)},
$$

which are weaker than the conditions $(2.17)$ on perturbations in the conventional definition (2.12).

To find the asymptotic symmetries of this extended class of spacetimes, let $\gamma_{a b}^{(\xi)}$ be the unphysical perturbation (2.18) generated by a diffeomorphism along $\xi^{a}$, as before. Imposing the requirements (3.7) we find that $\xi^{a}$ still satisfies the conditions (2.24) and (2.23). But since the unphysical metric is no longer universal at $\mathscr{I},\left.\gamma_{a b}^{(\xi)}\right|_{\mathscr{I}}$ is no longer required to vanish and so the condition (2.25) no longer holds. ${ }^{9}$ Using $\left.g^{a b} \gamma_{a b}^{(\xi)}\right|_{\mathscr{I}}=0$ we have instead

$$
£_{\xi} \bar{\varepsilon}_{a b c}=3 \alpha_{(\xi)} \bar{\varepsilon}_{a b c},
$$

where $\bar{\varepsilon}_{a b c}$ is the 3 -volume element $(3.1 \mathrm{~b})$ on $\mathscr{I}$.

Modding out by the trivial diffeomorphisms as before,${ }^{10}$ we thus find that the extended BMS algebra $\mathfrak{b}_{\text {ext }}[30]$ of $\Gamma_{\text {ext }}$ is generated by vector fields $\xi^{a}$ on $\mathscr{I}$ which are tangent to $\mathscr{I}$ and which satisfy

$$
\begin{aligned}
£_{\xi} n^{a} & =-\alpha_{(\xi)} n^{a}, \\
£_{\xi} \bar{\varepsilon}_{a b c} & =3 \alpha_{(\xi)} \bar{\varepsilon}_{a b c},
\end{aligned}
$$

where the function $\alpha_{(\xi)}$ is smooth and satisfies eq. (2.27). The structure of this extended BMS algebra can be analyzed as before. There is an infinite-dimensional abelian Lie ideal $\mathfrak{s}$ of supertranslations as before. However as a direct consequence of dropping eq. (2.26b) in favor of eq. $(3.9 \mathrm{~b})$, the factor algebra $\mathfrak{b}_{\text {ext }} / \mathfrak{s}$ is now isomorphic to the Lie algebra $\mathfrak{d i f f}\left(\mathbb{S}^{2}\right)$ of all smooth infinitesimal diffeomorphisms of $\mathbb{S}^{2}$. Hence we have $\mathfrak{b}_{\text {ext }}=\mathfrak{d i f f}\left(\mathbb{S}^{2}\right) \ltimes \mathfrak{s}$.

Thus, by weakening the universal structure near $\mathscr{I}-$ equivalently, extending the class of allowed perturbations - one obtains a bigger asymptotic symmetry algebra at null infinity which includes all the smooth diffeomorphisms of a 2 -sphere.

\section{The symplectic current of general relativity at null infinity}

In this section we evaluate the symplectic current of general relativity for the extended class of perturbations detailed in section 3. We will show that any choice of symplectic current, which is local and covariant, necessarily diverges in the limit to $\mathscr{I}$.

\footnotetext{
${ }^{9}$ The last condition in eq. (3.7) does not impose additional restrictions on $\xi^{a}$ at $\mathscr{I}$.

${ }^{10}$ The trivial vector fields are those for which the integral of symplectic current evaluated on $\delta \tilde{g}_{a b}$ and $£_{\xi} \tilde{g}_{a b}$ vanishes [8]. However, this quantity cannot be evaluated since the symplectic current diverges on $\mathscr{I}$, as we show in the next section. So we simply assume here that the trivial vector fields $\xi^{a}$ are those which vanish on $\mathscr{I}$, as for the standard definition of field configuration space. The symmetry algebra thus could change given a finite renormalized symplectic current; see section 5.2 below for further discussion of this point.
} 


\subsection{The symplectic current for general perturbations}

We briefly review the symplectic formalism for general relativity, though this formalism can be used for any local and covariant Lagrangian theory. The Lagrangian 4-form for vacuum general relativity is given by

$$
\boldsymbol{L}=\frac{1}{16 \pi} \tilde{\varepsilon}_{4} \tilde{R}
$$

where $\tilde{R}$ and $\tilde{\varepsilon}_{4} \equiv \tilde{\varepsilon}_{a b c d}$ are the Ricci scalar and the volume 4-form, respectively, in the physical spacetime. The variation of the Lagrangian is

$$
\delta \boldsymbol{L}=-\frac{1}{16 \pi} \tilde{G}^{a b} \delta \tilde{g}_{a b} \tilde{\varepsilon}_{4}+d \boldsymbol{\theta}(\tilde{g} ; \delta \tilde{g})
$$

where $\tilde{G}_{a b}$ is the physical Einstein tensor and the symplectic potential 3 -form $\boldsymbol{\theta}$ is given by $[8]$

$$
\begin{aligned}
\boldsymbol{\theta} \equiv \theta_{a b c} & =\frac{1}{16 \pi} \tilde{\varepsilon}_{d a b c} \tilde{v}^{d} \quad \text { with } \\
\tilde{v}^{a} & =\tilde{g}^{a e} \tilde{g}^{f h}\left[\tilde{\nabla}_{f} \tilde{\gamma}_{e h}-\tilde{\nabla}_{e} \tilde{\gamma}_{f h}\right] .
\end{aligned}
$$

Note that the symplectic potential (4.3) is not uniquely determined by the Lagrangian. We will investigate the ambiguities in the symplectic formalism in section 4.3.

The symplectic current 3 -form $\boldsymbol{\omega}$ is defined by ${ }^{11}$

$$
\boldsymbol{\omega}\left(\tilde{g} ; \delta_{1} \tilde{g}, \delta_{2} \tilde{g}\right)=\delta_{1} \boldsymbol{\theta}\left(\tilde{g} ; \delta_{2} \tilde{g}\right)-\delta_{2} \boldsymbol{\theta}\left(\tilde{g} ; \delta_{1} \tilde{g}\right)
$$

Using eq. (4.3), the symplectic current $\boldsymbol{\omega}$ for general relativity can be written as $\omega_{a b c}=$ $\tilde{\varepsilon}_{d a b c} \tilde{w}^{d} /(16 \pi)$ with $[8]$

$$
\begin{aligned}
\tilde{w}^{a} & =\tilde{P}^{a b c d e f} \tilde{\gamma}_{2 b c} \tilde{\nabla}_{d} \tilde{\gamma}_{1 e f}-[1 \leftrightarrow 2], \\
\tilde{P}^{a b c d e f} & =\tilde{g}^{a e} \tilde{g}^{f b} \tilde{g}^{c d}-\frac{1}{2} \tilde{g}^{a d} \tilde{g}^{b e} \tilde{g}^{f c}-\frac{1}{2} \tilde{g}^{a b} \tilde{g}^{c d} \tilde{g}^{e f}-\frac{1}{2} \tilde{g}^{b c} \tilde{g}^{a e} \tilde{g}^{f d}+\frac{1}{2} \tilde{g}^{b c} \tilde{g}^{a d} \tilde{g}^{e f},
\end{aligned}
$$

where " $[1 \leftrightarrow 2]$ " denotes the preceding expression with the labels 1 and 2 , labeling the perturbations, interchanged.

If the metric $\tilde{g}_{a b}$, the perturbation $\delta \tilde{g}_{a b}$ and the vector field $\xi^{a}$ generating an infinitesimal diffeomorphism satisfy some suitable asymptotic conditions at $\mathscr{I}$ so that the symplectic current $\boldsymbol{\omega}\left(\tilde{g} ; \delta \tilde{g}, £_{\xi} \tilde{g}\right)$ has a finite limit to $\mathscr{I}$, then the integral of this symplectic current over $\mathscr{I}$ can be used to define a generator of the symmetry corresponding to $\xi^{a}$ at null infinity [8]. Note that we require the full 3 -form $\boldsymbol{\omega}$ to have a limit to $\mathscr{I}$ and not just its pullback or integral over some surfaces that limit to $\mathscr{I}$; the latter procedure would, in general, depend on the choice of surfaces used and would not be covariant.

We now investigate whether the symplectic current has a limit to $\mathscr{I}$ when $\xi^{a}$ is a generator of the extended BMS algebra. To analyze the behavior of the symplectic current

\footnotetext{
${ }^{11}$ In general the integral of the symplectic current over a 3-dimensional surface will be degenerate and hence only defines a presymplectic form on the space of fields. We shall not be concerned with such issues and so continue to call $\boldsymbol{\omega}$ a symplectic current.
} 
at $\mathscr{I}$, it is convenient to express the symplectic current in terms of unphysical quantities which extend smoothly to $\mathscr{I}$ and are non-vanishing there in general. Thus we define

$$
\varepsilon_{a b c d}=\Omega^{4} \tilde{\varepsilon}_{a b c d}, \quad P^{a b c d e f}=\Omega^{-6} \tilde{P}^{a b c d e f}, \quad \gamma_{a b}=\Omega^{2} \tilde{\gamma}_{a b} .
$$

The relation between the physical derivative operator $\tilde{\nabla}$ and unphysical derivative operator $\nabla$ acting on any covector $v_{a}$ is given by

$$
\begin{aligned}
\tilde{\nabla}_{a} v_{b} & =\nabla_{a} v_{b}+C^{c}{ }_{a b} v_{c} \\
\text { with } \quad C_{a b}^{c} & =2 \Omega^{-1} \delta_{(a}^{c} n_{b)}-\Omega^{-1} n^{c} g_{a b}
\end{aligned}
$$

Inserting eq. (4.6) into eq. (4.5), using eq. (4.7), one obtains:

$$
\begin{aligned}
\boldsymbol{\omega} \equiv \omega_{a b c} & =\frac{1}{16 \pi} \varepsilon_{d a b c} w^{d}, \\
\text { with } \quad w^{a} & =\Omega^{-2} P^{a b c d e f} \gamma_{2 b c} \nabla_{d} \gamma_{1 e f}+\Omega^{-3} \gamma_{1}^{a b} n_{b} \gamma_{2 c}{ }^{c}-[1 \leftrightarrow 2] .
\end{aligned}
$$

Note that if both perturbations $\gamma_{1 a b}$ and $\gamma_{2 a b}$ are tangent to the standard field configuration space (2.12) and so satisfy the usual conditions (2.17), we have

$$
w^{a}=P^{a b c d e f} \tau_{2 b c} \nabla_{d} \tau_{1 e f}+\tau_{1}^{a b} \tau_{2 b}+\tau_{1}{ }^{a} \tau_{2 c}{ }^{c}-[1 \leftrightarrow 2]+O(\Omega),
$$

and in this case the symplectic current is finite at $\mathscr{I}$. In particular, when one of the perturbations is generated by an infinitesimal diffeomorphism corresponding to the BMS algebra, one can define the corresponding charges and fluxes following the procedure in [8].

\subsection{Divergence of the symplectic current on the extended phase space}

Now consider the case where one of the perturbations, say $\gamma_{1 a b}$, satisfies the usual set (2.17) of conditions, while the other one, $\gamma_{2 a b}$, lies in $\Gamma_{\text {ext }}$ and so satisfies only the weaker set of conditions (3.7). If in this case the symplectic current has a finite limit to $\mathscr{I}$ then one can hope to define charges and fluxes for the extended BMS algebra taking $\gamma_{2 a b}=\gamma_{a b}^{(\xi)}$ where $\left.\xi^{a}\right|_{\mathscr{I}}$ is an element in $\mathfrak{b}_{\text {ext }}$. However, as we now show, the symplectic current (4.8) in this case necessarily diverges in the limit to $\mathscr{I}$, unless $\gamma_{2 a b}$ also lies in the standard field configuration space $\Gamma_{0}$ [i.e. satisfies the standard conditions (2.17)], or the perturbation $\gamma_{1 a b}$ contains no gravitational radiation, i.e., has vanishing perturbed News tensor.

If the symplectic current $\boldsymbol{\omega}$ has a limit to $\mathscr{I}$ then its pullback to $\mathscr{I}$ will be proportional to $\bar{\varepsilon}_{3} n_{a} w^{a}$ where $\bar{\varepsilon}_{3} \equiv \bar{\varepsilon}_{a b c}$ is the 3 -volume element on $\mathscr{I}$. It will suffice for our purposes to show that $n_{a} w^{a}$ does not have a limit to $\mathscr{I}$. Using eqs. (2.16), (2.15) for $\gamma_{1 a b}$ and eqs. (3.4) for $\gamma_{2 a b}$ we have

$$
\begin{aligned}
n_{a} w^{a}= & \Omega^{-1}\left[n_{a} P^{a b c d e f} \gamma_{2 b c} \nabla_{d} \tau_{1 e f}-n_{a} P^{a b c d e f} \tau_{1 b c} \nabla_{d} \gamma_{2 e f}\right] \\
& +\chi_{2 a} \tau_{1}{ }^{a}+\frac{1}{2} \sigma_{2} \tau_{1}{ }^{a} n_{a}-\frac{3}{2} \Omega^{-1} n^{a} \chi_{2 a} \tau_{1 b}{ }^{b}-\frac{1}{2} \Omega^{-2} n_{a} n^{a} \gamma_{2}{ }^{b c} \tau_{1 b c}+\frac{1}{2} \Omega^{-1} n_{a} n^{a} \sigma_{2} \tau_{1 b}{ }^{b} .
\end{aligned}
$$

Due to eqs. (2.9) and (3.7) the second line in eq. (4.10) has a finite limit to $\mathscr{I}$. Now if $n_{a} w^{a}$ has a finite limit to $\mathscr{I}$ then we must have $\lim _{\rightarrow \mathscr{I}} \Omega n_{a} w^{a}=0$. From eq. (4.10) we have

$$
\Omega n_{a} w^{a}=n_{a} P^{a b c d e f} \gamma_{2 b c} \nabla_{d} \tau_{1 e f}-n_{a} P^{a b c d e f} \tau_{1 b c} \nabla_{d} \gamma_{2 e f}+O(\Omega)
$$


where $O(\Omega)$ indicates terms which vanish in the limit to $\mathscr{I}$. Evaluating the first term of eq. (4.11) at $\mathscr{I}$, using eq. (3.7), we have

$$
\begin{aligned}
\left.n_{a} P^{a b c d e f} \gamma_{2 b c} \nabla_{d} \tau_{1 e f}\right|_{\mathscr{I}} & =n^{a} \gamma_{2}{ }^{b c} \nabla_{c} \tau_{1 a b}-\frac{1}{2} n^{a} \gamma_{2}{ }^{b c} \nabla_{a} \tau_{1 b c} \\
& =-\frac{1}{2} \gamma_{2}{ }^{b c} n^{a} \nabla_{a} \tau_{1 b c} .
\end{aligned}
$$

Similarly for the second term in eq. (4.11) we have (from eqs. (2.17), (3.7))

$$
\left.n_{a} P^{a b c d e f} \tau_{1 b c} \nabla_{d} \gamma_{2 e f}\right|_{\mathscr{I}}=-\frac{1}{2} \tau_{1}^{b c} n^{a} \nabla_{a} \gamma_{2 b c}=0 .
$$

Thus eq. (4.11) can be written as

$$
\Omega n_{a} w^{a}=-\frac{1}{2} \gamma_{2}^{a b} n^{c} \nabla_{c} \tau_{1 a b}+O(\Omega) .
$$

We now simplify the above expression further using the vacuum Einstein equation. Perturbing eq. (2.4), for the perturbation $\gamma_{1 a b}$ we have (see also eq. (67) of [8])

$$
\left.\delta_{1} S_{a b}\right|_{\mathscr{I}}=2 n_{a} \tau_{1 b}+2 n_{b} \tau_{1 a}-n^{c} \nabla_{c} \tau_{1 a b}-n^{c} \tau_{1 c} g_{a b} .
$$

Using eq. (4.15) in eq. (4.14) to eliminate the derivative of $\tau_{1 a b}$, and eqs. (2.17), (3.7) we get

$$
\Omega n_{a} w^{a}=\frac{1}{2} \gamma_{2}^{a b} \delta_{1} S_{a b}+O(\Omega) .
$$

Further, since $\gamma_{2}{ }^{a b}$ is tangent to $\mathscr{I}$ (from eq. (3.4)) we can replace $S_{a b}$ by its pullback to $\mathscr{I} \bar{S}_{a b}$ to get

$$
\Omega n_{a} w^{a}=\frac{1}{2} \gamma_{2}^{a b} \delta_{1} \bar{S}_{a b}+O(\Omega)
$$

Now for a asymptotically flat spacetime the News tensor on $\mathscr{I}$ is defined by

$$
N_{a b}=\bar{S}_{a b}-\rho_{a b}
$$

where $\rho_{a b}$ is the unique symmetric tensor field on $\mathscr{I}$ constructed from the (usual) universal structure at $\mathscr{I}$ in Theorem 5 of [5]. Thus, for the perturbation $\gamma_{1 a b}$ we have $\delta_{1} \rho_{a b}=0$ and we can replace $\delta_{1} \bar{S}_{a b}$ in eq. (4.17) with $\delta_{1} N_{a b}$ to get

$$
\Omega n_{a} w^{a}=\frac{1}{2} \gamma_{2}^{a b} \delta_{1} N_{a b}+O(\Omega)
$$

For general perturbations $\gamma_{1 a b}$ the perturbed News $\delta_{1} N_{a b}$ does not vanish on $\mathscr{I}$, indicating the presence of (linearized) gravitational radiation, although it is subject to the constraints

$$
g^{a b} \delta_{1} N_{a b}=0, \quad n^{a} \delta_{1} N_{a b}=0 .
$$

If the quantity (4.19) vanishes for all perturbations $\gamma_{1 a b}$, then $\gamma_{2 a b}$ must be of the form $\alpha g_{a b}+n_{(a} v_{b)}+O(\Omega)$, but it then follows from eqs. (3.7) that $\gamma_{2 a b}=O(\Omega)$. 
We therefore conclude that

1. The symplectic current has a finite limit to $\mathscr{I}$ for all perturbations $\gamma_{1 a b}$ that are tangent to the standard phase space $\Gamma_{0}$, if and only if $\left.\gamma_{2 a b}\right|_{\mathscr{I}}=0$, that is, $\gamma_{2 a b}$ also is tangent to $\Gamma_{0}$. In particular when $\gamma_{2 a b}=\gamma_{a b}^{(\xi)}$ is a perturbation generated by an infinitesimal diffeomorphism $\xi^{a}$, then $\left.\gamma_{a b}^{(\xi)}\right|_{\mathscr{I}}=0$ and thus $\left.\xi^{a}\right|_{\mathscr{I}}$ is an element of the usual BMS algebra $\mathfrak{b}$ (see eq. (2.25)).

2. The symplectic current has a finite limit to $\mathscr{I}$ for any $\gamma_{2 a b}=\gamma_{a b}^{(\xi)}$ generated by an infinitesimal diffeomorphism $\xi^{a}$ in $\mathfrak{b}_{\text {ext }}$ which is not in $\mathfrak{b}$, if and only if $\gamma_{1 a b}$ has vanishing perturbed News, that is, $\gamma_{1 a b}$ is non-radiating at $\mathscr{I}$.

We emphasize that we have shown that (except in the cases discussed above) the limit to $\mathscr{I}$ of the symplectic current $\boldsymbol{\omega}$ as a 3 -form does not exist. That is, the symplectic current diverges as we approach any point of $\mathscr{I}$ along any curve in the unphysical spacetime independently of any choice of coordinates.

We now compare our result with the procedure used by Campiglia and Laddha [30], who obtained finite charges associated with generators of the extended algebra. Their procedure can be described as follows. In the physical spacetime pick some Bondi coordinate system $\left(r, u, x^{A}\right)$ near $\mathscr{I}$. Consider the surfaces $\Sigma_{t}$ given by $t=u+r=$ constant and integrate the symplectic current $\boldsymbol{\omega}$ on $\Sigma_{t}$ with the perturbation $\delta_{2} \tilde{g}_{a b}=£_{\xi} \tilde{g}_{a b}$ generated by some diffeomorphism in $\mathfrak{b}_{\text {ext }}$ and $\delta_{1} \tilde{g}_{a b}$ lying in $\Gamma_{0}$. This integral can be rewritten as an integral over a 2 -sphere of $u=$ constant on $\Sigma_{t}$. Then as $u \rightarrow-\infty$ this integral diverges linearly in $u$ if the vector field $\xi^{a}$ is an element of $\mathfrak{b}_{\text {ext }}$ which is not in the usual BMS algebra $\mathfrak{b}$. To get a finite symplectic form for all symmetries in $\mathfrak{b}_{\text {ext }}$, ref. [30] then imposes the boundary condition $C_{A B} \sim 1 / u^{1+\epsilon}$ along every $\Sigma_{t}$ where $C_{A B}$ is a subleading piece of the physical metric on the 2 -spheres in Bondi coordinates. The symplectic form on $\mathscr{I}$ is then defined as the $t \rightarrow \infty$ limit of this symplectic form on the surfaces $\Sigma_{t}$.

We note that, in contrast to our approach, the procedure used by [30] is not covariant. In particular their boundary condition $C_{A B} \sim 1 / u^{1+\epsilon}$ along every $\Sigma_{t}$ is not invariant under supertranslations (this was noted also by [30]). Thus, if this condition holds in one choice of Bondi coordinate system, it fails to hold in another Bondi coordinate system related to the first by a supertranslation. Similarly, this condition fails to hold if one instead integrates the symplectic current on some different family of surfaces which are supertranslated relative to their choice of $\Sigma_{t}$. We also note that for the "soft charge" on $\mathscr{I}$ defined in $[29,30]$ to be finite one needs to impose $C_{A B} \sim 1 /|u|^{1+\epsilon}$ along $\mathscr{I}$ as $u \rightarrow \pm \infty$ (the "soft charge" vanishes for elements of the Lorentz algebra $\mathfrak{s o}(1,3)$ upon integration over the 2-spheres and this restriction is not required). As is well-known [16], this implies that the memory effect in such spacetimes must vanish which is a severe restriction on the class of spacetimes.

\subsection{Ambiguities in the symplectic current}

As shown in the previous section, the symplectic current of a perturbation in the standard phase space $\Gamma_{0}$ with any element of the extended BMS algebra (which is not in the usual BMS algebra) is not finite at $\mathscr{I}$. However, the symplectic current of general relativity is 
not uniquely determined by its Lagrangian, and it was claimed in [28] that the symplectic current can be made finite at $\mathscr{I}$ by a suitable choice of such an ambiguity. Since the computations of [28] are tied to a Bondi coordinate system, it is not apparent if their choice of the ambiguity is local and covariant. In this section we show that any ambiguity in the symplectic current, which is local and covariant cannot be used to make the symplectic current finite at $\mathscr{I}$, in general.

The symplectic potential $\boldsymbol{\theta}$ defined by eq. (4.2) is ambiguous up to the addition of a local and covariant 3 -form $\boldsymbol{X}(\tilde{g} ; \delta \tilde{g})$ which is linear in $\delta \tilde{g}$ and closed, i.e. $d \boldsymbol{X}=0$. It can be shown quite generally [37] that such a closed form must be exact i.e. $\boldsymbol{X}(\tilde{g} ; \delta \tilde{g})=d \boldsymbol{Y}(\tilde{g} ; \delta \tilde{g})$ for some 2-form $\boldsymbol{Y}$ which is local and covariant and linear in $\delta \tilde{g}$. Thus, the ambiguity in the symplectic potential is

$$
\boldsymbol{\theta}(\tilde{g} ; \delta \tilde{g}) \mapsto \boldsymbol{\theta}(\tilde{g} ; \delta \tilde{g})+d \boldsymbol{Y}(\tilde{g} ; \delta \tilde{g})
$$

From eq. (4.4), the corresponding ambiguity in the symplectic current is given by ${ }^{12}$

$$
\boldsymbol{\omega}\left(\tilde{g} ; \delta_{1} \tilde{g}, \delta_{2} \tilde{g}\right) \mapsto \boldsymbol{\omega}\left(\tilde{g} ; \delta_{1} \tilde{g}, \delta_{2} \tilde{g}\right)+d\left[\boldsymbol{Z}\left(\tilde{g} ; \delta_{1} \tilde{g}, \delta_{2} \tilde{g}\right)-\boldsymbol{Z}\left(\tilde{g} ; \delta_{2} \tilde{g}, \delta_{1} \tilde{g}\right)\right]
$$

where, for later convenience, we have defined the 2 -form

$$
\boldsymbol{Z}\left(\tilde{g} ; \delta_{1} \tilde{g}, \delta_{2} \tilde{g}\right)=\delta_{1} \boldsymbol{Y}\left(\tilde{g} ; \delta_{2} \tilde{g}\right),
$$

Note that it follows from eq. (4.23) that any such $\boldsymbol{Z}$ must satisfy the condition

$$
\delta_{3} \boldsymbol{Z}\left(\tilde{g} ; \delta_{1} \tilde{g}, \delta_{2} \tilde{g}\right)-\delta_{1} \boldsymbol{Z}\left(\tilde{g} ; \delta_{3} \tilde{g}, \delta_{2} \tilde{g}\right)=0
$$

for arbitrary perturbations $\delta_{3} \tilde{g}$ of the metric, even those that do not lie in $\Gamma_{0}$.

We first define the notion of a scaling dimension for tensors following [34]. A tensor $L^{a \ldots}{ }_{b \ldots}$ with $u$ upper and $l$ lower indices constructed out of the unphysical metric $g_{a b}$ and the conformal factor $\Omega$ is said to have a scaling dimension $s$, if under a scaling of the conformal factor $\Omega \mapsto \lambda \Omega$ and the metric $g_{a b} \mapsto \lambda^{2} g_{a b}$ by a constant $\lambda$, we have $L^{a \ldots} b_{\ldots} \mapsto \lambda^{s-u+l} L^{a \ldots} b_{b .}$. Note that the scaling dimension is independent of the tensor index positions and is additive under tensor products. One sees from this that the relevant scaling dimensions are:

$$
\Omega: 1, \quad g_{a b}: 0, \quad \varepsilon_{a b c d}: 0, \quad \nabla:-1, \quad n_{a}: 0
$$

Since we are interested in the behavior of $\boldsymbol{Z}$ near $\mathscr{I}$, it is useful to write everything in terms of unphysical quantities which are smooth at $\mathscr{I}$. Since $\boldsymbol{Z}\left(\delta_{1} \tilde{g}, \delta_{2} \tilde{g}\right)$ is linear in both physical metric perturbations, in terms of the unphysical perturbations $\gamma_{1 a b}, \gamma_{2 a b}$ we must have

$$
\boldsymbol{Z}\left(\gamma_{1}, \gamma_{2}\right)=\sum_{p, q} \boldsymbol{W}^{a b c d e_{1} \ldots e_{p} f_{1} \ldots f_{q}}\left(\nabla_{e_{1}} \cdots \nabla_{e_{p}} \gamma_{1 a b}\right)\left(\nabla_{f_{1}} \cdots \nabla_{f_{q}} \gamma_{2 c d}\right)
$$

where $p$ and $q$ (each ranging from 0 to some finite value) count the number of derivatives of $\gamma_{1 a b}$ and $\gamma_{2 a b}$, respectively. Here $\boldsymbol{W}^{a b c d e_{1} \ldots e_{p} f_{1} \ldots f_{q}}$ are some local and covariant tensorvalued 2-forms which are local functionals of the unphysical metric, the unphysical Riemann

\footnotetext{
${ }^{12}$ Note that the equations of motion are unaffected by the change $\boldsymbol{L} \mapsto \boldsymbol{L}+d \boldsymbol{K}$ in the Lagrangian. This does not affect the symplectic current since $\delta_{1} \delta_{2} \boldsymbol{K}-\delta_{2} \delta_{1} \boldsymbol{K}=0$.
} 
tensor and its derivatives and the conformal factor. The scaling dimension of $\gamma_{1 a b}$ and $\gamma_{2 a b}$ is 0 , and since the scaling dimension of the symplectic potential $\boldsymbol{\theta}$ is -3 it follows from eq. (4.21) that the scaling dimension of $\boldsymbol{Z}$ is -2 . Therefore, the scaling dimension of $\boldsymbol{W}^{a b c d e} e_{1} \ldots e_{p} f_{1} \ldots f_{q}$ is $-2+p+q$.

Now we analyze the possible forms of $\boldsymbol{W}^{a b c d e_{1} \ldots e_{p} f_{1} \ldots f_{q}}$ that can appear in eq. (4.26). Note that our goal is to find a $\boldsymbol{Z}$ that can get rid of the divergence in the symplectic current in the limit to $\mathscr{I}$. From eq. (4.19) we see that this diverging term depends analytically on the background unphysical metric. Thus, in any candidate expression for $\boldsymbol{Z}$ of the form (4.26), we can assume that $\boldsymbol{W}^{a b c d e_{1} \ldots e_{p} f_{1} \ldots f_{q}}$ is an analytic functional of its arguments. ${ }^{13}$ Using the Einstein equation (2.4), we can eliminate the covariant derivatives of $n_{a}$ in favor of $S_{a b}$ and its derivatives. Similarly, the unphysical Riemann tensor and its derivatives can be rewritten in terms of $S_{a b}$ and the Weyl tensor $C_{a b c d}$ and their derivatives using

$$
R_{a b c d}=C_{a b c d}+g_{a[c} S_{d] b}-g_{b[c} S_{d] a}
$$

Thus any typical term in $\boldsymbol{W}^{\text {abcde } e_{1} \ldots e_{p} f_{1} \ldots f_{q}}$ can be schematically written in the form ${ }^{14}$

$$
\Omega^{v} \prod_{i=1}^{r}(\nabla)^{s_{i}} S_{a b} \prod_{j=1}^{u}(\nabla)^{t_{j}}\left(C_{a b c d}\right) \times(\text { terms with } 0 \text { scaling dimension })
$$

where we have suppressed contractions with the metric $g_{a b}$ for simplicity of notation. In the above $r, u \geq 0$ count the number of factors involving $S_{a b}$ and $C_{a b c d}$ respectively and $s_{i}, t_{j} \geq 0$ count the number of derivatives occurring in each such term. Note that $v$ is allowed to be negative. Comparing the scaling dimensions of eq. (4.28) and $\boldsymbol{W}^{a b c d e e_{1} \ldots e_{p} f_{1} \ldots f_{q}}$ gives

$$
-2+p+q=v-\sum_{i}^{r} s_{i}-2 r-\sum_{j}^{u} t_{j}-2 u .
$$

From the above we see that $v \geq-2$. Lets consider the "most singular" term where $v=-2$, and thus $p=q=r=u=0$; this term does not contain any $S_{a b}$ or $C_{a b c d}$ and has no derivatives of the perturbations $\gamma_{1 a b}, \gamma_{2 a b}$. Then, eq. (4.26) simplifies to the form

$$
\boldsymbol{Z}\left(\gamma_{1}, \gamma_{2}\right)=\boldsymbol{W}^{a b c d} \gamma_{1 a b} \gamma_{2 c d}+O\left(\Omega^{-1}\right)
$$

where $\boldsymbol{W}^{a b c d}=\Omega^{-2} \times$ (terms with 0 scaling dimension). Recall that when $\gamma_{1 a b}$ is a perturbation in $\Gamma_{0}$ we have $\gamma_{1 a b}=\Omega \tau_{1 a b}$ where $\tau_{1 a b}$ is smooth and non-vanishing, in general, at $\mathscr{I}$. In this case, the "most singular" term we have considered in eq. (4.30) diverges as $\Omega^{-1}$ near $\mathscr{I}$. This is precisely the term one would need to cancel the diverging part of the symplectic current in eq. (4.19).

\footnotetext{
${ }^{13}$ We emphasize that $\boldsymbol{W}^{a b c d e_{1} \ldots e_{p} f_{1} \ldots f_{q}}$ being analytic in its functional dependence is unrelated to the analyticity of the unphysical metric on the spacetime manifold. We do not impose any analyticity conditions on the spacetimes under consideration.

${ }^{14}$ Note that, by the peeling theorem (Theorem 11 [5]), for an asymptotically-flat spacetime, $C_{a b c d}$ vanishes and $\Omega^{-1} C_{a b c d}$ has a finite limit at $\mathscr{I}$. Thus, in eq. (4.28) we can use $\Omega^{-1} C_{a b c d}$ instead; this only changes the last term in eq. (4.29) to $-3 u$ and does not affect the rest of the argument. We use the Weyl tensor $C_{a b c d}$ since we allow the background spacetime to satisfy some "extended" notion of asymptotic flatness for which the peeling theorem might not hold.
} 
Let us now figure out what the 2 -form $\boldsymbol{W}^{a b c d}$ can be. Notice that $\Omega$ can only appear with a power -2 in the expression for $\boldsymbol{W}^{a b c d}$, in particular, any terms with 0 scaling dimension that we need cannot be constructed by multiplying some powers of $\Omega$ with something with a negative scaling dimension. Since $\boldsymbol{W}^{\text {abcd }}$ must be local and covariant the only quantities available are $g_{a b}, \varepsilon_{a b c d}$ and $n_{a}$ - note that any derivatives of these will have negative scaling dimension. Using eqs. (2.9) and (3.7) leads to just two possible terms which appear at order $\Omega^{-2}$, in terms of which we can write eq. (4.30) as

$$
\boldsymbol{Z}\left(\gamma_{1}, \gamma_{2}\right) \equiv Z_{a b}\left(\gamma_{1}, \gamma_{2}\right)=\Omega^{-2}\left(A \varepsilon_{a b}^{c d}+B \delta_{[a}^{c} \delta_{b]}^{d}\right) g^{e f} \gamma_{1 c e} \gamma_{2 d f}+O\left(\Omega^{-1}\right)
$$

where $A, B$ are some constants. Since we have only computed the $\boldsymbol{Z}$ up to terms of $O\left(\Omega^{-1}\right)$, our consistency condition eq. (4.24), must also hold to this order. However, it is easy to verify that eq. (4.31) fails to satisfy this condition since $\left.\delta_{3} g_{a b}\right|_{\mathscr{I}} \neq 0$ for an arbitrary perturbation in the extended class of perturbations. That is, there does not exist an ambiguity $\boldsymbol{Y}$ in the symplectic potential such that eq. (4.31) is of the form eq. (4.23). Thus we conclude that any choice of the symplectic current for general relativity, which is local and covariant, must diverge in the limit to $\mathscr{I}$, in general, when at least one of the perturbations is taken to be in the extended class of allowed perturbations.

\section{Other issues}

Since our covariance arguments are not airtight, as discussed in the Introduction, we now consider some other arguments for and against the extension of the BMS algebra. We focus on two specific issues: the desirability of having a definition of Bondi 4-momentum and the freedom in choosing a field configuration space.

\subsection{Existence of Bondi four-momentum}

The standard BMS algebra $\mathfrak{b}$ contains a preferred four dimensional subalgebra of translations, associated with the existence of Bondi 4-momentum. By contrast, the extended algebra $\mathfrak{b}_{\text {ext }}$ does not, as we show explicitly in appendix B. Therefore, there is no natural universal definition of Bondi 4-momentum in any context where $\mathfrak{b}_{\text {ext }}$ is the asymptotic symmetry algebra. This lack of a definition of Bondi 4-momentum would seem to be a difficulty for any physical interpretation of the extended algebra.

However, the notion of Bondi 4-momentum would still apply in the context of the symmetry algebra $\mathfrak{b}_{\text {ext }}$, but in a solution-dependent manner. Specifically, given a solution $\left(M, g_{a b}, \Omega\right)$, one can define the field configuration space (2.12) associated with that solution, and from it obtain an associated translation subgroup of $\mathfrak{b}_{\text {ext }}$ and corresponding 4-momentum charge. The 4-momenta associated with two different solutions need not be comparable, as in general they would lie in different spaces. This status of 4-momentum in the extended algebra would be analogous to the status of angular momentum in the standard BMS context. There, stationary solutions determine preferred Poincaré subalgebras of the BMS algebra, with associated linear and angular momentum charges, but the angular momentum charges associated with two different stationary solutions need not be comparable as they live in different spaces. 


\subsection{Choice of field configuration space}

In the body of the paper we considered an enlargement of the field configuration space $\Gamma_{0}$ to a larger space $\Gamma_{\text {ext }}$ which contains additional unphysical metrics $\left(M, g_{a b}, \Omega\right)$ that are related to metrics already in $\Gamma_{0}$ by diffeomorphisms and conformal transformations. This raises the question of what criterion can one use to define field configuration spaces in general? How much gauge (here diffeomorphism and conformal) freedom can or should be fixed?

A key consideration is that the phase space of the theory is constructed from the field configuration space $\Gamma_{0}$ or $\Gamma_{\text {ext }}$ by modding out by degeneracy directions of the presymplectic form $[8,36,38]$. The construction of the symmetry algebra also mods out by these degeneracies (see footnote 10 above). The degeneracy directions correspond to gauge transformations (diffeomorphism or conformal) which vanish sufficiently rapidly near the boundary. Therefore, in defining the initial field configuration space, it should not matter how much gauge freedom is fixed, since any residual gauge freedom will be removed in the construction of the final phase space and symmetry algebra. However, one must be careful that one fixes only "true gauge" degrees of freedom, that is, degeneracy directions of the presymplectic form.

The question then is whether the standard configuration space $\Gamma_{0}$ of eq. (2.12) has already fixed some degrees of freedom which are physical and not gauge (i.e. do not correspond to degeneracy directions of the presymplectic form). Unfortunately, it is not straightforward to answer this question, since as we have shown, for the relevant metric perturbations the presymplectic current is either divergent on $\mathscr{I}$, or if one uses the renormalized presymplectic current of ref. [28], the presymplectic form may or may not be covariant. If we suppose for the sake of argument that it is covariant, then an examination of eq. (5.27) of ref. [28] shows that the presymplectic form on the extended phase space does not exhibit any degeneracy directions. This would argue in favor of the extended algebra.

\section{Discussion and conclusions}

Using the definition of asymptotically-flat spacetimes we showed how the BMS algebra $\mathfrak{b}$ arises as the asymptotic symmetry algebra at null infinity, emphasizing the role of the smoothness and topological assumptions in the definition. A crucial role in our analysis was played by the fact that the conformal class of metrics on a 2 -sphere is unique up to diffeomorphisms. This can be used to show that the conformal factor $\Omega$ and the unphysical metric $\left.g_{a b}\right|_{\mathscr{I}}$ can be chosen to be universal in the class of asymptotically-flat spacetimes, without loss of generality.

We then considered an extended class of spacetimes where $\left.g_{a b}\right|_{\mathscr{I}}$ is not considered universal as proposed by Campiglia and Laddha [30]. In this class of spacetimes, the asymptotic symmetry algebra includes all smooth diffeomorphisms of a 2-sphere. We showed, however, that the symplectic current of general relativity evaluated on such extended symmetries does not have a finite limit to null infinity, and that no local and covariant ambiguity in the choice of symplectic current cures this divergent behavior. This suggests that the extension of the BMS symmetry algebra proposed by [29, 30] is ill-defined on the phase space at null infinity. However, as discussed in the introduction, a possible loophole is the 
fact that imposing locality and covariance at all stages of the computation may be too strong a restriction, and instead one should only impose covariance on the symplectic form obtained by integrating the symplectic current.

\section{Acknowledgments}

This work is supported in part by the NSF grants PHY-1404105 and PHY-1707800 to Cornell University.

\section{A Metric on $\mathscr{I}$ and conformal factor in a neighborhood can be chosen to be universal}

In this appendix, we prove the following property of asymptotically flat spacetimes. Suppose that we are given two different unphysical spacetimes $\left(M, g_{a b}, \Omega\right)$ and $\left(M^{\prime}, g_{a b}^{\prime}, \Omega^{\prime}\right)$ corresponding to two different physical spacetimes. By specializing to a neighborhood of $\mathscr{I}$ and using a diffeomorphism we can identify the background manifolds. In addition, by exploiting the diffeomorphism freedom $\left(g_{a b}, \Omega\right) \rightarrow\left(\psi_{*} g_{a b}, \psi_{*} \Omega\right)$ and the conformal rescaling freedom $\left(g_{a b}, \Omega\right) \rightarrow\left(\omega^{2} g_{a b}, \omega \Omega\right)$, we can without loss of generality take

$$
\Omega=\Omega^{\prime}
$$

in a neighborhood of $\mathscr{I}$, as well as

$$
\left.g_{a b}\right|_{\mathscr{I}}=\left.g_{a b}^{\prime}\right|_{\mathscr{I}}
$$

together with the Bondi condition

$$
\left.\nabla_{a} \nabla_{b} \Omega\right|_{\mathscr{I}}=0 .
$$

Thus, without loss of generality, we can take the conformal factor in a neighborhood of $\mathscr{I}$ and the unphysical metric evaluated on $\mathscr{I}$ to be universal, the same for all asymptotically flat spacetimes.

The proof is based on constructing suitable coordinate systems in a neighborhood of $\mathscr{I}$. Let us pick any asymptotically-flat physical spacetime $\left(\tilde{M}, \tilde{g}_{a b}\right)$ and let $\left(M, g_{a b}\right)$ be its conformal completion with some choice of conformal factor $\Omega$ satisfying the Bondi condition (2.8). Pick any cross-section $S \cong \mathbb{S}^{2}$ of $\mathscr{I}$ and consider the induced metric $q_{a b}$ at $S$. Since the conformal class of metrics on $\mathbb{S}^{2}$ is unique up to diffeomorphisms, there exists a unit round 2-metric $s_{a b}$ and a smooth positive function $\varpi$ on $S$ so that $q_{a b}=\varpi^{2} s_{a b}$ on $S$. We can now use the freedom (2.10) in the choice of conformal factor at $S$ to make $\varpi=1$, so that $q_{a b}=s_{a b}$ on $S$.

Next, we choose coordinates $x^{A}=\left(x^{1}, x^{2}\right)=(\theta, \varphi)$ on $S$ so that this 2-metric $s_{A B}$ takes the standard form

$$
s_{A B} d x^{A} d x^{B}=d \theta^{2}+\sin ^{2} \theta d \varphi^{2} .
$$

We extend the coordinates $x^{A}$ to all of $\mathscr{I}$ by imposing the condition

$$
\left.n^{a} \nabla_{a} x^{A}\right|_{\mathscr{I}}=0 .
$$


We also define the function $u$ on $\mathscr{I}$ by the conditions $\left.u\right|_{S}=0$ and

$$
n^{a} \nabla_{a} u=1 .
$$

This defines the coordinate system $\left(u, x^{A}\right)$ on $\mathscr{I}$. Note that on $\mathscr{I}, £_{n} q_{a b}=0$ (eq. (2.11)) and thus in our choice of conformal factor we have that the induced metric takes the form (A.4) on all of $\mathscr{I}$.

We next define spacetime coordinates $\left(\Omega, u, x^{A}\right)$ in a neighborhood of $\mathscr{I}$. First, since $\left.\Omega\right|_{\mathscr{I}}=0$ and $\left.\nabla_{a} \Omega\right|_{\mathscr{I}} \neq 0$, we can use $\Omega$ as a coordinate. Next, we extend the coordinates $\left(u, x^{A}\right)$ away from $\mathscr{I}$. There are many different choices of extension. The extension we choose here leads to the familiar Bondi coordinates in the physical spacetime (see also [39]). Consider a family of null hypersurfaces transverse to $\mathscr{I}$ which intersect $\mathscr{I}$ in the crosssections $S_{u}$ given by $u=$ constant. In a sufficiently small neighborhood of $\mathscr{I}$, such null hypersurfaces generate a null foliation. We first extend the coordinate $u$ by demanding that it be constant along these null hypersurfaces. We define

$$
l_{a}=\nabla_{a} u,
$$

the null normal to these hypersurfaces, which satisfies $l^{a} l_{a}=0$ and $\left.l^{a} n_{a}\right|_{\mathscr{I}}=1$ from the condition (A.6). Then, we extend the angular coordinates $x^{A}$ to a neighborhood of $\mathscr{I}$ by demanding $l^{a} \nabla_{a} x^{A}=0$.

Finally, we specialize the definition of $\Omega$ off $\mathscr{I}$ as follows. To extend $\Omega$ away from $\mathscr{I}$ we use the freedom in the conformal factor away from $\mathscr{I}$ to demand that the 2 -spheres of constant $u$ and $\Omega$ have the same area element as the unit sphere, that is, if $h_{A B}$ is the 2 -metric on the surfaces of constant $u$ and $\Omega$ then we demand that $\operatorname{det} h=\operatorname{det} s$ in the $x^{A}$-coordinates. This fixes $\Omega$ uniquely away from $\mathscr{I}$. Thus we have set up a conformal Bondi coordinate system $\left(\Omega, u, \theta^{A}\right)$ in a neighborhood of $\mathscr{I}$ in which the unphysical metric takes the form

$$
d s^{2} \equiv-W e^{2 \beta} d u^{2}+2 e^{2 \beta} d \Omega d u+h_{A B}\left(d x^{A}-U^{A} d u\right)\left(d x^{B}-U^{B} d u\right)
$$

where $W, \beta, h_{A B}$, and $U^{A}$ are smooth functions of the coordinates $\left(\Omega, u, x^{A}\right)$. Note that the metric components $g_{\Omega \Omega}$ and $g_{\Omega A}$ vanish due to $l^{a} l_{a}=l^{a} \nabla_{a} x^{A}=0$. Now if we assume that the metric components in eq. (A.8) have an asymptotic expansion in integer powers of $\Omega$ near $\mathscr{I}$, then from the construction of our coordinates, and using the condition (2.9) we have

$$
\begin{aligned}
& W=O\left(\Omega^{2}\right), \quad \beta=O(\Omega) \quad U^{A}=O(\Omega), \\
& h_{A B}=s_{A B}+\Omega C_{A B}+O\left(\Omega^{2}\right) \quad s^{A B} C_{A B}=0 .
\end{aligned}
$$

If we further define $r=\Omega^{-1}$ then we get the familiar Bondi coordinate form ${ }^{15}$ for the physical metric $\tilde{g}_{a b}=\Omega^{-2} g_{a b}=r^{2} g_{a b}$. Note that the asymptotic falloffs in $r$ used for the physical metric in Bondi coordinates follow from eq. (A.9) and are a direct consequence of asymptotic flatness and the construction of the coordinate system.

\footnotetext{
${ }^{15}$ The usual Bondi coordinate expression incorporates two other conditions, $W=\Omega^{2}+O\left(\Omega^{3}\right), U^{A}=$ $O\left(\Omega^{2}\right)$, that are obtained by imposing the Einstein equations with the assumption (2.1) on the stress energy tensor.
} 
Since $\left.n^{a} \nabla_{a} u\right|_{\mathscr{I}}=1$, the unphysical metric $g_{a b}$ at $\mathscr{I}$ in these coordinates is

$$
\left.g_{a b}\right|_{\mathscr{I}} \equiv 2 d \Omega d u+s_{A B} d x^{A} d x^{B} .
$$

We now identify all asymptotically-flat spacetimes with each other, in a neighborhood of $\mathscr{I}$, by identifying their points in the coordinates $\left(\Omega, u, x^{A}\right)$ constructed above. With this choice it follows that the conformal factor $\Omega$ and the unphysical metric (A.10) are universal i.e. independent of the chosen asymptotically-flat physical spacetime. Different asymptoticallyflat physical metrics change only the subleading metric components in eq. (A.9) but agree to leading order.

We emphasize that many of the choices made in constructing the Bondi coordinates are irrelevant to this argument and are made just for convenience. For instance, the choice of the unit-metric $s_{a b}$ is irrelevant. In any asymptotically-flat spacetime, we can instead choose the coordinates $x^{A}$ and use the freedom $\omega$ in the conformal factor at $\mathscr{I}$ so that $q_{a b}=q_{a b}^{(0)}$ where $q_{a b}^{(0)}$ is any fixed metric on $\mathbb{S}^{2}$. Then we can proceed with the rest of the construction as before to conclude that $\left.g_{a b}\right|_{\mathscr{I}}$ is universal. The only important ingredient is the fact that $\mathbb{S}^{2}$ has a unique conformal class of metrics up to diffeomorphisms. Similarly, the extension of the coordinates away from $\mathscr{I}$ can also be chosen differently. For instance, consider the null vector field $\left.l^{a}\right|_{\mathscr{I}} \equiv \partial / \partial \Omega$ transverse to the cross-sections $S_{u}$ of $\mathscr{I}$. Instead of choosing $\Omega$ away from $\mathscr{I}$ to make the 2 -spheres have unit area, we can extend this vector field away from $\mathscr{I}$ by demanding that $l^{a} \equiv \partial / \partial \Omega$ be an affinely-parameterized null vector field i.e. $l^{a} l_{a}=0$ and $l^{b} \nabla_{b} l^{a}=0$. Then we can extend the coordinates $\left(u, x^{A}\right)$ by parallel transport along $l^{a}$. This defines a conformal Gaussian null coordinate system in a neighborhood of $\mathscr{I}[16,35]$. Now we can again identify all the asymptotically-flat spacetimes in these conformal Gaussian null coordinates to conclude that $\Omega$ and $\left.g_{a b}\right|_{\mathscr{I}}$ are universal in a neighborhood of $\mathscr{I}$. We can also develop an asymptotic expansion for the metric components in this coordinate system similar to eqs. (A.8) and (A.9) (see [35]) and again see that different asymptotically-flat physical metrics only change the subleading metric components but agree to leading order.

In conclusion, given the definition of asymptotic flatness and that the conformal class of metrics on $\mathbb{S}^{2}$ is unique, it is always possible to identify all the unphysical spacetimes in a neighborhood of $\mathscr{I}$ so that $\Omega$ and $\left.g_{a b}\right|_{\mathscr{I}}$ are independent of the physical spacetime under consideration.

\section{B The extended BMS algebra does not contain any preferred translation subalgebra}

In this appendix we show that the extended BMS algebra does not contain any preferred subalgebra (i.e. a Lie ideal) of translations. Since the asymptotic symmetry algebra is common to all spacetimes under consideration, its Lie bracket is independent of the choice of background spacetime. Thus we can compute the Lie bracket on any choice of background spacetime, and in particular we can take the background physical spacetime to be Minkowski. Let us choose a conformal completion for Minkowski so that the induced 
metric $q_{a b}$ on $\mathscr{I}$ is that of a unit-metric on $\mathbb{S}^{2}$ and let $\mathscr{D}$ denote the covariant derivative of $q_{a b}$. Let $u$ be an affine parameter along the null geodesics of $n^{a}$ so that $\left.n^{a} \nabla_{a} u\right|_{\mathscr{I}}=1$.

From eq. (3.9), any element $\xi^{a}$ of the algebra $\mathfrak{b}_{\text {ext }}$ can be written as

$$
\xi^{a}=X^{a}+\frac{1}{2}\left(u-u_{0}\right) \mathscr{D}_{b} X^{b} n^{a}+f^{\prime} n^{a}
$$

where $f^{\prime}$ is any function on $\mathbb{S}^{2}$ (representing a supertranslation), $X^{a}$ is a vector field on $\mathbb{S}^{2}$ while the function $\alpha_{(\xi)}=\frac{1}{2} \mathscr{D}_{a} X^{a}$. The Lie bracket of a supertranslation $f n^{a} \in \mathfrak{s}$ and $\xi^{a}$ is then

$$
[f n, \xi]^{a}=\beta n^{a} \quad \text { where } \beta=-X^{a} \mathscr{D}_{a} f+\frac{1}{2} \mathscr{D}_{a} X^{a} f
$$

It is straightforward to check that $£_{n} \beta=0$ and so $\beta n^{a}$ is a supertranslation in $\mathfrak{s}$.

If translations are a Lie ideal in $\mathfrak{b}_{\text {ext }}$ then $\beta n^{a}$ would also be a translation whenever $f n^{a}$ is translation. To investigate this we proceed as follows. Since $f n^{a}$ is a translation, $f$ is a $\ell=$ 0,1 spherical harmonic on $\mathbb{S}^{2}$ - it is well-known that the limit of translations in Minkowski spacetime to $\mathscr{I}$ are precisely such vector fields. Let $X^{a}$ be a $\ell^{\prime}$-vector harmonic so that

$$
X^{a}=\mathscr{D}^{a} F+\varepsilon^{a b} \mathscr{D}_{b} G
$$

for some functions $F$ and $G$ which are $\ell^{\prime}$-spherical harmonics. In the case $\xi^{a}$ is an element of the BMS algebra $\mathfrak{b}$ so that $X^{a}$ is an element of the Lorentz algebra $\mathfrak{s o}(1,3)$, both $F$ and $G$ are spherical harmonics with $\ell^{\prime}=1$. The function $F$ corresponds to Lorentz boosts while $G$ corresponds to Lorentz rotations. When $\xi^{a}$ is an element of the extended BMS algebra $\mathfrak{b}_{\text {ext }}, \ell^{\prime} \geq 1$ and then $\ell^{\prime}>1$ modes of $F$ and $G$ can be thought of as "extended" boosts and rotations.

Using the decomposition eq. (B.3) in eq. (B.2) we have

$$
\beta=-\mathscr{D}^{a} F \mathscr{D}_{a} f+\frac{1}{2} \mathscr{D}^{2} F f+\varepsilon^{a b} \mathscr{D}_{a} G \mathscr{D}_{b} f
$$

Now we wish to find the spherical harmonic mode $L$ of $\beta$ when $f$ is a translation i.e. $\ell=0,1$-harmonic mode while the harmonic mode of $F$ and $G$ can be $\ell^{\prime} \geq 1$. It is useful to consider the following different cases.

Case 1: $f$ is time translation, $\ell=0$. Then,

$$
\beta=\frac{1}{2} \mathscr{D}^{2} F f=-\frac{1}{2} \ell^{\prime}\left(\ell^{\prime}+1\right) F f
$$

so

$$
\mathscr{D}^{2} \beta=-\ell^{\prime}\left(\ell^{\prime}+1\right) \beta=-L(L+1) \beta
$$

Thus, $\beta=0$ if $F=0$ else $\beta$ is a $L=\ell^{\prime}$ mode. When $X^{a} \in \mathfrak{s o}(1,3), \ell^{\prime}=1$ and this can be interpreted as the fact that a time translation is invariant under Lorentz rotations given by $G$ but changes by a spatial translation under Lorentz boosts given by $F$. 
Case 2: $f$ is spatial translation i.e. $\ell=1, F=0$ and $G \neq 0$. Then we have,

$$
\beta=\varepsilon^{a b} \mathscr{D}_{a} G \mathscr{D}_{b} f
$$

and

$$
\begin{aligned}
\mathscr{D}^{2} \beta & =\left[-\ell^{\prime}\left(\ell^{\prime}+1\right)-\ell(\ell+1)+2\right] \beta+2 \varepsilon^{a b} \mathscr{D}_{c} \mathscr{D}_{a} G \mathscr{D}^{c} \mathscr{D}_{b} f \\
& =-\ell^{\prime}\left(\ell^{\prime}+1\right) \beta=-L(L+1) \beta
\end{aligned}
$$

where in the last line we use $\ell=1$ and that $\mathscr{D}_{a} \mathscr{D}_{b} f=-q_{a b} f$ for such functions. Thus, $\beta$ is a $L=\ell^{\prime}$ mode. Thus, when $X^{a} \in \mathfrak{s o}(1,3), \ell^{\prime}=1$, a spatial translation changes by another spatial translation under Lorentz rotations given by $G$.

Case 3: $f$ is spatial translation i.e $\ell=1, F \neq 0$ and $G=0$.

$$
\beta=-\mathscr{D}_{a} F \mathscr{D}_{a} f+\frac{1}{2} \mathscr{D}^{2} F f
$$

To find the $L$-mode of $\beta$, we multiply the above equation with the (complex conjugate) spherical harmonic $\bar{Y}_{L, M}$ and integrate over $\mathbb{S}^{2}$ to get (we have left the area element of the unit-metric on $\mathbb{S}^{2}$ implicit for notational convenience)

$$
\int \beta \bar{Y}_{L, M}=-\int \mathscr{D}^{a} F \mathscr{D}_{a} f \bar{Y}_{L, M}-\frac{1}{2} \ell^{\prime}\left(\ell^{\prime}+1\right) \int F f \bar{Y}_{L, M}
$$

The first term on the right-hand-side can be rewritten using repeated integration-by-parts as

$$
\begin{aligned}
-\int \mathscr{D}^{a} F \mathscr{D}_{a} f \bar{Y}_{L, M} & =\int F \mathscr{D}^{2} f \bar{Y}_{L, M}+\int F \mathscr{D}_{a} f \mathscr{D}^{a} \bar{Y}_{L, M} \\
& =\int F \mathscr{D}^{2} f \bar{Y}_{L, M}-\int \mathscr{D}_{a} F f \mathscr{D}^{a} \bar{Y}_{L, M}-\int F f \mathscr{D}^{2} \bar{Y}_{L, M} \\
& =\int F \mathscr{D}^{2} f \bar{Y}_{L, M}+\int \mathscr{D}^{2} F f \bar{Y}_{L, M}-\int F f \mathscr{D}^{2} \bar{Y}_{L, M}+\int \mathscr{D}_{a} F \mathscr{D}^{a} f \bar{Y}_{L, M} \\
-\int \mathscr{D}^{a} F \mathscr{D}_{a} f \bar{Y}_{L, M} & =\frac{1}{2} \int F \mathscr{D}^{2} f \bar{Y}_{L, M}+\frac{1}{2} \int \mathscr{D}^{2} F f \bar{Y}_{L, M}-\frac{1}{2} \int F f \mathscr{D}^{2} \bar{Y}_{L, M} \\
& =\frac{1}{2}\left[-\ell(\ell+1)-\ell^{\prime}\left(\ell^{\prime}+1\right)+L(L+1)\right] \int F f \bar{Y}_{L, M}
\end{aligned}
$$

Thus, we have

$$
\int \beta \bar{Y}_{L, M}=\left[-\frac{1}{2} \ell(\ell+1)-\ell^{\prime}\left(\ell^{\prime}+1\right)+\frac{1}{2} L(L+1)\right] \int F f \bar{Y}_{L, M}
$$

Expanding the functions $F$ and $f$ in terms of the corresponding spherical harmonics $Y_{\ell^{\prime}, m^{\prime}}$ and $Y_{\ell, m}$ respectively, we can write the final integral in terms of the $3 j$-symbols (see section 34 [40]) (or in terms of the Clebsch-Gordon coefficients, section 3.7 [41]) as

$$
\int Y_{\ell^{\prime}, m^{\prime}} Y_{\ell, m} \bar{Y}_{L, M}=(-1)^{M} \sqrt{\frac{(2 \ell+1)\left(2 \ell^{\prime}+1\right)(2 L+1)}{4 \pi}}\left(\begin{array}{ccc}
\ell, & \ell^{\prime}, & L \\
0, & 0, & 0
\end{array}\right)\left(\begin{array}{ccc}
\ell, & \ell^{\prime}, & L \\
m, & m^{\prime}, & -M
\end{array}\right)
$$


Since $f$ is a spatial translation with $\ell=1$, we have

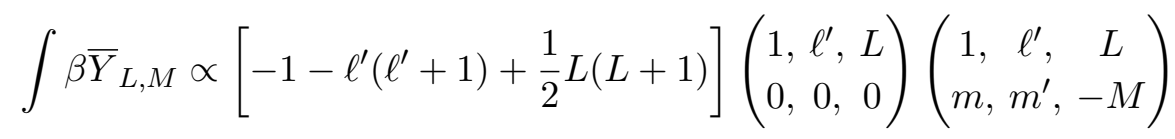

where we have ignored non-zero constant factors. The right-hand-side is non-vanishing if and only if (section 34 [40])

$$
\begin{array}{r}
-1-\ell^{\prime}\left(\ell^{\prime}+1\right)+\frac{1}{2} L(L+1) \neq 0 \\
1+\ell^{\prime}+L \quad \text { is even } \\
\ell^{\prime}-1 \leq L \leq \ell^{\prime}+1, \quad M=m+m^{\prime}
\end{array}
$$

These conditions on $L$ can be satisfied if and only if (we do not need the conditions on $M$ for our argument)

$$
L=\left\{\begin{array}{lll}
0 & \text { for } \ell^{\prime}=1 \\
\ell^{\prime}-1 & \text { or } \ell^{\prime}+1 & \text { for } \ell^{\prime} \geq 2
\end{array}\right.
$$

Note that for the $\ell^{\prime}=1$ case, the value $L=\ell^{\prime}+1=2$ is ruled out by the first condition in eq. (B.15). Thus, when $X^{a} \in \mathfrak{s o}(1,3), \ell^{\prime}=1$, a spatial translation changes by a time translation under Lorentz boosts given by $F$.

For the usual BMS algebra $\mathfrak{b}$ with $X^{a} \in \mathfrak{s o}(1,3)$ and $\ell^{\prime}=1$, we see that in each case $\beta$ is a spherical harmonic with $L=0,1$ that is $\beta n^{a}$ is a translation. Thus the translation subalgebra is preserved under the Lie bracket of $\mathfrak{b}$ i.e. there is a preferred 4-dimensional Lie ideal of translations in $\mathfrak{b}$. For the extended BMS algebra $\mathfrak{b}_{\text {ext }}$ with $X^{a} \in \mathfrak{d i f f}\left(\mathbb{S}^{2}\right)$ and $\ell^{\prime} \geq 2$, the translations $f n^{a}$, in general, change by $\beta n^{a}$ where $\beta$ contains a spherical harmonic as high as $L=\ell^{\prime}+1$. Thus, translations are not preserved by the Lie bracket of $\mathfrak{b}_{\text {ext }}$ and are not a preferred subalgebra (Lie ideal) of $\mathfrak{b}_{\text {ext }}$. The above argument can be generalized to show that there is, in fact, no finite-dimensional Lie ideal of extended BMS algebra.

The absence of a preferred translation algebra poses a problem for the prescription used by [30] to define a symplectic form on $\mathscr{I}$. As discussed above, the boundary condition imposed by [30] near spatial infinity to obtain a finite symplectic form for $\mathfrak{b}_{\text {ext }}$ is not invariant under general supertranslations, but is invariant under translations in a specific choice of Bondi coordinates. However, as we have shown, there is no preferred notion of pure translations in $\mathfrak{b}_{\text {ext }}$. Thus, the translation invariance of the boundary condition in [30] is also unclear.

Open Access. This article is distributed under the terms of the Creative Commons Attribution License (CC-BY 4.0), which permits any use, distribution and reproduction in any medium, provided the original author(s) and source are credited.

\section{References}

[1] H. Bondi, M.G.J. van der Burg and A.W.K. Metzner, Gravitational waves in general relativity. 7. Waves from axisymmetric isolated systems, Proc. Roy. Soc. Lond. A 269 (1962) 21 [INSPIRE]. 
[2] R.K. Sachs, Gravitational waves in general relativity. 8. Waves in asymptotically flat space-times, Proc. Roy. Soc. Lond. A 270 (1962) 103 [InSPIRE].

[3] R. Sachs, Asymptotic symmetries in gravitational theory, Phys. Rev. 128 (1962) 2851 [INSPIRE].

[4] R. Penrose, Zero rest mass fields including gravitation: asymptotic behavior, Proc. Roy. Soc. Lond. A 284 (1965) 159 [INSPIRE].

[5] R. Geroch, Asymptotic structure of space-time, in Asymptotic structure of space-time, F.P. Esposito and L. Witten eds., Plenum Press, New York, NY, U.S.A. (1977) [InSPIRE].

[6] R.P. Geroch and J. Winicour, Linkages in general relativity, J. Math. Phys. 22 (1981) 803 [INSPIRE].

[7] A. Ashtekar and M. Streubel, Symplectic geometry of radiative modes and conserved quantities at null infinity, Proc. Roy. Soc. Lond. A 376 (1981) 585 [inSPIRE].

[8] R.M. Wald and A. Zoupas, A general definition of 'conserved quantities' in general relativity and other theories of gravity, Phys. Rev. D 61 (2000) 084027 [gr-qc/9911095] [INSPIRE].

[9] T. He, V. Lysov, P. Mitra and A. Strominger, BMS supertranslations and Weinberg's soft graviton theorem, JHEP 05 (2015) 151 [arXiv:1401.7026] [INSPIRE].

[10] A. Strominger and A. Zhiboedov, Gravitational memory, BMS supertranslations and soft theorems, JHEP 01 (2016) 086 [arXiv:1411.5745] [INSPIRE].

[11] D. Kapec, V. Lysov, S. Pasterski and A. Strominger, Higher-dimensional supertranslations and Weinberg's soft graviton theorem, Ann. Math. Sci. Appl. 02 (2017) 69 [arXiv: 1502.07644] [INSPIRE].

[12] S.G. Avery and B.U.W. Schwab, Burg-Metzner-Sachs symmetry, string theory and soft theorems, Phys. Rev. D 93 (2016) 026003 [arXiv:1506.05789] [INSPIRE].

[13] M. Campiglia and A. Laddha, Asymptotic symmetries of gravity and soft theorems for massive particles, JHEP 12 (2015) 094 [arXiv: 1509.01406] [INSPIRE].

[14] A. Campoleoni, D. Francia and C. Heissenberg, On higher-spin supertranslations and superrotations, JHEP 05 (2017) 120 [arXiv:1703.01351] [INSPIRE].

[15] S. Pasterski, A. Strominger and A. Zhiboedov, New gravitational memories, JHEP 12 (2016) 053 [arXiv: 1502 .06120] [INSPIRE].

[16] S. Hollands, A. Ishibashi and R.M. Wald, BMS supertranslations and memory in four and higher dimensions, Class. Quant. Grav. 34 (2017) 155005 [arXiv:1612.03290] [INSPIRE].

[17] P. Mao and H. Ouyang, Note on soft theorems and memories in even dimensions, Phys. Lett. B 774 (2017) 715 [arXiv: 1707.07118] [InSPIRE].

[18] M. Pate, A.-M. Raclariu and A. Strominger, Gravitational memory in higher dimensions, JHEP 06 (2018) 138 [arXiv: 1712.01204] [INSPIRE].

[19] A. Chatterjee and D.A. Lowe, BMS symmetry, soft particles and memory, Class. Quant. Grav. 35 (2018) 094001 [arXiv: 1712.03211] [INSPIRE].

[20] S.W. Hawking, M.J. Perry and A. Strominger, Soft hair on black holes, Phys. Rev. Lett. 116 (2016) 231301 [arXiv:1601.00921] [INSPIRE].

[21] A. Strominger, Black hole information revisited, World Scientific, Singapore (2019), pg. 109 [arXiv: 1706.07143] [INSPIRE]. 
[22] S.W. Hawking, M.J. Perry and A. Strominger, Superrotation charge and supertranslation hair on black holes, JHEP 05 (2017) 161 [arXiv:1611.09175] [INSPIRE].

[23] A. Strominger, Lectures on the infrared structure of gravity and gauge theory, arXiv: 1703.05448 [INSPIRE].

[24] F. Cachazo and A. Strominger, Evidence for a new soft graviton theorem, arXiv:1404.4091 [INSPIRE].

[25] G. Barnich and C. Troessaert, Symmetries of asymptotically flat 4 dimensional spacetimes at null infinity revisited, Phys. Rev. Lett. 105 (2010) 111103 [arXiv:0909.2617] [INSPIRE].

[26] G. Barnich and C. Troessaert, Aspects of the BMS/CFT correspondence, JHEP 05 (2010) 062 [arXiv: 1001.1541] [INSPIRE].

[27] D. Kapec, V. Lysov, S. Pasterski and A. Strominger, Semiclassical Virasoro symmetry of the quantum gravity S-matrix, JHEP 08 (2014) 058 [arXiv: 1406.3312] [INSPIRE].

[28] G. Compère, A. Fiorucci and R. Ruzziconi, Superboost transitions, refraction memory and super-Lorentz charge algebra, JHEP 11 (2018) 200 [arXiv:1810.00377] [INSPIRE].

[29] M. Campiglia and A. Laddha, Asymptotic symmetries and subleading soft graviton theorem, Phys. Rev. D 90 (2014) 124028 [arXiv:1408.2228] [INSPIRE].

[30] M. Campiglia and A. Laddha, New symmetries for the gravitational S-matrix, JHEP 04 (2015) 076 [arXiv: 1502.02318] [INSPIRE].

[31] L. Freidel, F. Hopfmüller and A. Riello, Asymptotic renormalization in flat space: symplectic potential and charges of electromagnetism, JHEP 10 (2019) 126 [arXiv:1904.04384] [INSPIRE].

[32] R.M. Wald, General relativity, The University of Chicago Press, Chicago, IL, U.S.A. (1984) [INSPIRE].

[33] L. Bieri, Solutions of the Einstein vacuum equations, in Extensions of the stability theorem of the Minkowski space in general relativity, AMS/IP Stud. Adv. Math. 45, (2009).

[34] S. Hollands and A. Ishibashi, Asymptotic flatness and Bondi energy in higher dimensional gravity, J. Math. Phys. 46 (2005) 022503 [gr-qc/0304054] [INSPIRE].

[35] S. Hollands and A. Thorne, Bondi mass cannot become negative in higher dimensions, Commun. Math. Phys. 333 (2015) 1037 [arXiv:1307.1603] [INSPIRE].

[36] D. Harlow and J.-Q. Wu, Covariant phase space with boundaries, arXiv:1906.08616 [INSPIRE].

[37] R.M. Wald, On identically closed forms locally constructed from a field, J. Math. Phys. 31 (1990) 2378.

[38] J. Lee and R.M. Wald, Local symmetries and constraints, J. Math. Phys. 31 (1990) 725 [INSPIRE].

[39] L.A. Tamburino and J.H. Winicour, Gravitational fields in finite and conformal Bondi frames, Phys. Rev. 150 (1966) 1039 [InSPIRE].

[40] F.W.J. Olver et al. eds., NIST digital library of mathematical functions, release 1.0.18, http://dlmf.nist.gov/, 27 March 2018.

[41] J.J. Sakurai, Modern quantum mechanics, Addison-Wesley Publishing Company, U.S.A. (1994). 